Provided for non-commercial research and education use. Not for reproduction, distribution or commercial use.

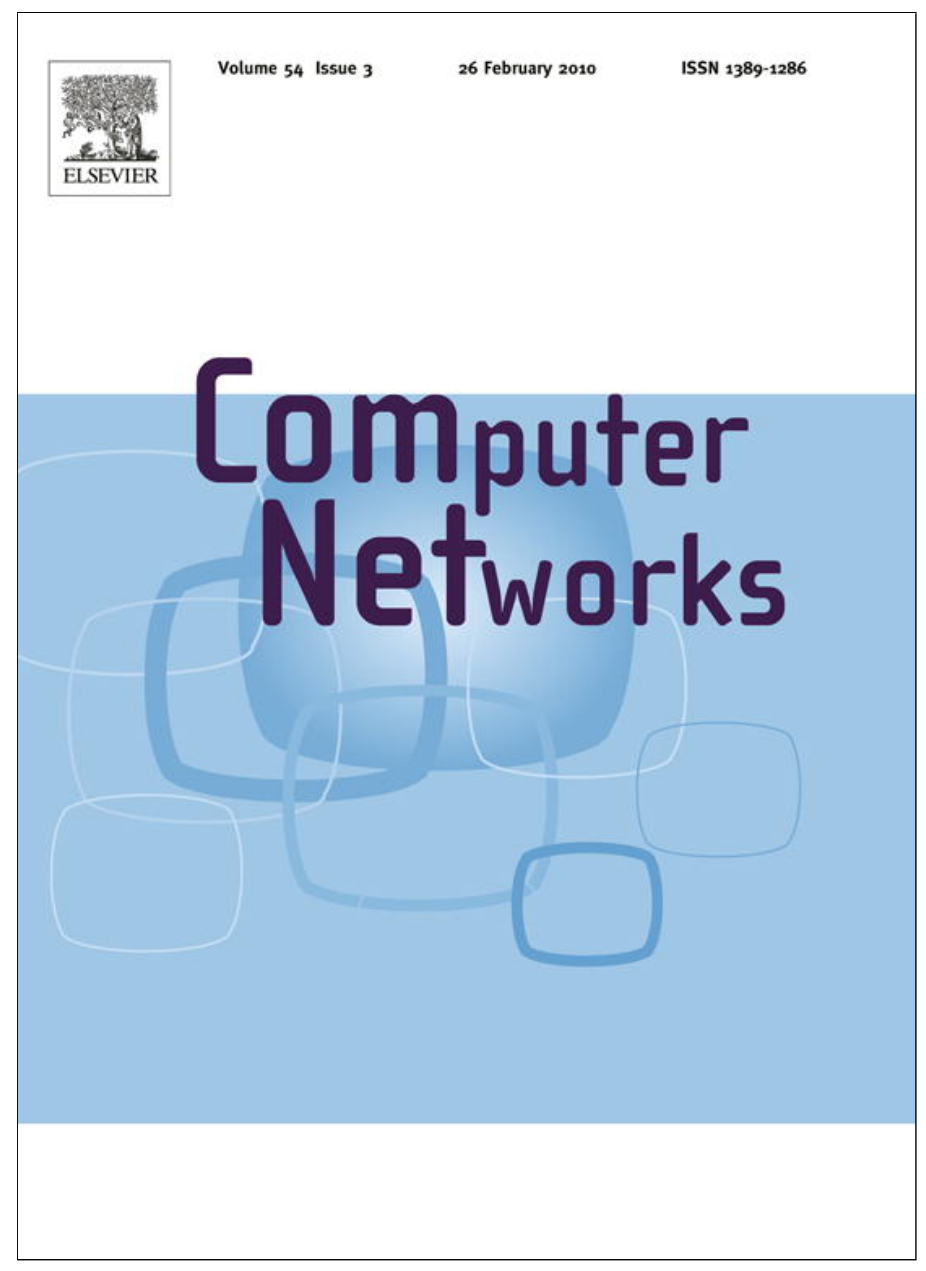

This article appeared in a journal published by Elsevier. The attached copy is furnished to the author for internal non-commercial research and education use, including for instruction at the authors institution and sharing with colleagues.

Other uses, including reproduction and distribution, or selling or licensing copies, or posting to personal, institutional or third party websites are prohibited.

In most cases authors are permitted to post their version of the article (e.g. in Word or Tex form) to their personal website or institutional repository. Authors requiring further information regarding Elsevier's archiving and manuscript policies are encouraged to visit:

http://www.elsevier.com/copyright 


\title{
A new economic generalized particle model for flow control
}

\author{
Xiang Feng ${ }^{\mathrm{a}, *}$, Francis C.M. Lau ${ }^{\mathrm{b}}$ \\ ${ }^{a}$ Department of Computer Science and Engineering, East China University of Science and Technology, China \\ bepartment of Computer Science, The University of Hong Kong, Hong Kong
}

\section{A R T I C L E I N F O}

\section{Article history:}

Received 29 October 2008

Accepted 23 September 2009

Available online 29 September 2009

Responsible Editor: I.F. Akyildiz

\section{Keywords:}

Intelligent bandwidth allocation

Economic generalized particle model

(EGPM)

Price and demands dynamic modulation

Distributed and parallel algorithm

Dynamical process

Computer networks

\begin{abstract}
A B S T R A C T
The problem of bandwidth allocation in computer networks can be likened to the supplydemand problem in economics. This paper presents the economic generalized particle model (EGPM) approach to intelligent allocation of network bandwidth. EGPM is a significant extension and further development of the generalized particle model (GPM) [1]. The approach comprises two major components: (1) dynamic allocation of network bandwidth based on GPM; and (2) dynamic modulation of price and demands of network bandwidth. The resulting algorithm can be easily implemented in a distributed fashion. Pricing being the network control mechanism in EGPM is carried out by a tatonnement process. We discuss the EGPM's convergence and show that the approach is efficient in achieving the global Pareto optimum. Via simulations, we test the approach, analyze its parameters and compare it with GPM and a genetic-algorithm-based solution.
\end{abstract}

(c) 2009 Elsevier B.V. All rights reserved.

\section{Introduction}

\subsection{Approaches to the bandwidth allocation problem}

Well-known optimization approaches that have been applied to network resource allocation include: the maxmin fairness (progressive filling) algorithm [2], Lagrangian multiplier approaches such as the ones by Kelly and Low et al. [3-9], the ant colony optimization approach [10-12], and genetic algorithms [13-15].

The max-min fairness algorithm has been widely used in digital networks, where it is used to allot bandwidth as equally as possible to all the users under certain transmission conditions. Although the algorithm is easy to realize, it tends to yield lower utilization of bandwidth resources than other approaches. The algorithms proposed by Kelly and Low et al. dynamically control the data transmission rates of source nodes in the network so that the global utility of all the source nodes may be maximized.

\footnotetext{
* Corresponding author. Address: Meilong Road 130, Shanghai 200237, PR China. Tel.: +862164253471 103.

E-mail address: xfeng@ecust.edu.cn (X. Feng).
}

These algorithms can usually achieve a comparatively higher utilization of network resources with some degree of fairness. Using these algorithms, however, flow control is centralized, which makes the algorithms difficult to realize in real environments. Evolutionary algorithms (EA) are heuristic-based global search and optimization methods that have found their way into virtually every area of real world optimization. Ant colony optimization (ACO) and genetic algorithm (GA) are well-known examples; they belong to the class of meta-heuristics or approximate algorithms capable of obtaining fairly good solutions to hard combinatorial optimization problems in a reasonable amount of computation time. Their main limitation is that their empirical performance is unknown, and they could sometimes consume excessive amounts of computation time.

During the past several years, a number of approaches using economic models for resource allocation have been proposed, which include [16-21]. Some of them divide the traffic into multiple priority classes, and use a fixed price for each class. To achieve greater network's efficiency, a better strategy may be to use dynamic pricing whose variation depends on network activities such as 
congestion. Wang and Schulzrinne [16] proposed a strategy where the price depends on the service class's average demand. The price is negotiable through a negotiation protocol. The strategy however requires resource reservation which obviously would incur some amount of overhead. In the pricing model of Baglietto et al. [17], the same amount of bandwidth is assigned to all traffic classes. Although simple, this approach may not be that reasonable as different customers may have different demands for bandwidth. Pricing in the scheme proposed in [18] is governed by a well-defined statistical model based on the source traffic. The scheme however does not take into account dynamic changes in the traffic.

Auction-based algorithms could be an effective model for solving classical assignment problems. Bitsaki et al. [19] pointed out that auctioning as an approach can outperform its main competitors by a margin for some important types of problems, and is also well suited for parallel implementation.

Pricing as an effective means to achieve economic efficiency for computer networks has attracted much attention recently. A number of pricing schemes have been proposed, such as $[20,21]$. An appropriate pricing policy will provide incentives for users to behave in a way that would improve the overall utilization and performance of the network. A proper pricing policy influences the users' demands and the network revenue. There exist many papers on pricing for communication networks using the principles of microeconomics. Kelly et al., for example, use congestion price for rate control in wired networks, where elastic traffic users for throughput reasons can dynamically change their data rates [4]. Siris adopts a similar approach for CDMA networks [22]. Low presented a duality model of several TCP/AQM gate protocols [6], which treats these protocols as distributed primal-dual algorithms carried out over the Internet in real-time to maximize the aggregate utility and subject to certain capacity constraints. Kelly et al.'s and Low's methods can be regarded as directly or indirectly based on the Lagrangian multiplier method, and because of that, flow control is centralized.

\subsection{The generalized particle model (GPM)}

Recently, Shuai and Feng proposed the generalized particle model (GPM) approach [1] $]^{1}$ which is based on hybrid energy functions. GPM can overcome some of the main deficiencies of the other approaches while retaining some of the good features of those well-known approaches just discussed. GPM was shown to be easy to realize, able to achieve comparatively higher network resource availability, and suitable for distributed implementation. GPM uses two "force-fields", the demand force-field and the resource force-field. There are numerous particles and forces in every force-field, where the particles and forces follow their own dynamic equations to represent the network entities and their behaviors and interactions respectively. The approach

\footnotetext{
1 The work reported in [25] predated that of [1]; the latter incorporated several major improvements and achieved better results for the bandwidth allocation problem.
}

features high parallelism, low computational complexity, and simplicity for hardware implementation. The GPM approach, however, has the following limitations when applied to the network bandwidth allocation problem.

- The demand force-field in GPM is used to determine the pricing. Since the prices of bandwidth computed based on the demand force-field are linked to many factors such as congestion, supply and demands of bandwidth, interaction of various network entities, etc., the convergence rate to the equilibrium prices is low.

- Since many parameters in GPM need to be correctly chosen, GPM may not always produce a solution for efficient resource utilization. In order to accommodate as many connections as possible in a congested network, the resources need to be used efficiently. GPM's complex solutions (in both pricing and resource allocation) may be impractical although theoretically they can achieve better resource utilization.

- When the network traffic changes dynamically, the pricing is modulated via several or more iterative steps, and only when these steps are completely over can the new price take effect. Thus, prices modulate slowly in response to changes of the network traffic.

\subsection{Economic generalized particle model (EGPM)}

This paper presents the EGPM approach for network bandwidth allocation. EPGM is based on GPM [1] and the excess demand function [28]. EGPM retains the advantages and overcome the disadvantages of the price-based approaches mentioned above, as follows.

- It adds congestion-dependent components to the price.

- It takes network activities and dynamic traffic changes into account.

- Its pricing depends on the excess demands of network resources.

- It does not require resource reservation.

- It uses price to reflect the dynamic situation of resource demands and the supply.

- It does not assign the same amount of bandwidth to all classes of traffic.

- Its complexity is relatively low.

Similar to the auction algorithm, EGPM rests on the belief that an appropriate pricing policy will provide incentives for the users to behave in ways that would improve the overall utilization and performance. EGPM makes use of the following ideas in the field of economics.

\subsubsection{Yield management}

By adjusting the allocation of (limited) resources, a company can optimize its total revenue or "yield." In [23], it is suggested that (1) it is expensive or impossible to store excess resources; (2) commitments need to be made when future demand is uncertain; (3) the company should differentiate among customers who may have different demand curves; (4) the same unit of capacity can be used to deliver many different products or services; 
and (5) producers are profit-oriented and have absolute freedom of action. Obviously, the bandwidth allocation problem has all these characteristics.

\subsubsection{General equilibrium model}

General equilibrium theory seeks to explain production, consumption and prices in an economy. Walras suggests that equilibrium can be achieved through a process of tatonnement [24].

\subsubsection{Tatonnement process}

Prices are lowered for goods with positive prices and excess supply, and should be raised for goods with excess demand [24]. EGPM applies the tatonnement process to GPM for the pricing of bandwidth.

\subsubsection{Pareto efficiency}

If an economic system is Pareto efficient, then no individual can be made better off without another individual being made worse off [24]. In this paper, we prove that the solution obtained by the EGPM algorithm is globally Pareto optimal.

We study particularly the relationship between price and demands in networks, and present the detailed EGPM approach which exploits the price-demands trade-off in solving the bandwidth allocation problem.

The following summarizes the differences of EGPM from GPM.

- In EGPM, the resource force-field is retained for allocating network resources; but instead of the demand forcefield, the economic tatonnement process is used to determine the prices.

- The solution obtained by the EGPM algorithm is globally Pareto optimal.

- The EGPM algorithm is simpler than GPM in terms of the initial conditions and the choice of the main parameters in the algorithm, hence more practical.

- By the tatonnement process, EGPM is more effective in resource utilization than GPM. Also, EGPM's convergence rate is faster.

- In EGPM, only the value of the excess demand function is used in updating the price; hence, price modulation works faster than GPM in response to dynamic network traffic changes.

The provision of network services can be viewed as an economy where users are consumers and network resources are commodities. The price for bandwidth dynamically reflects the equilibrium between demand and supply. When the supply of bandwidth is less than the demands of bandwidth, the network becomes congested, and the price will rise; whereas when the supply of bandwidth is more than the demands of bandwidth, the price will fall. In EGPM, prices depend on the traffic demands and the available bandwidth. The bandwidth allocated to a user is equal to the demand for a price that the user can accept. Hence, the pricing scheme in EGPM is dynamic where the prices are not fixed and can change as the traffic load in the network changes.
In this paper, we consider the idealized situation where users can freely choose their shares of fixed or variable bandwidth in order to maximize their benefit. The network coordinates their choices through resource pricing. By bringing up the price at times of peak usage of bandwidth, the demands would adjust themselves, and the result is that less capacity is needed to meet the demands, and the capital utilization becomes higher. Some traditional approaches would reserve certain capacity for the users ahead of time. Reservation however neglects the efficiency gains that are possible through sharing of resources. EGPM avoids reservation so as to take advantage of statistical multiplexing.

To avoid large communication overheads in the computation, we (1) price only bandwidth (but not buffers), and (2) avoid reservation. In typical network situations, both bandwidth and buffer capacities will eventually become scarce. We believe that scarcity is best treated through pricing as price-based allocation is consistent with a competitive marketplace. We consider bandwidth to be a natural commodity to price, whereas pricing buffers is less satisfactory from a user's point of view. Furthermore, network performance is usually more sensitive to bandwidth than to buffers.

The EGPM algorithm is divided into two parts, as shown in Fig. 1: (1) dynamic allocation of network bandwidth based on GPM, and (2) dynamic modulation of prices and demands based on the tatonnement process. The tatonnement process and auctioning share some common features. Allocation based on GPM can realize the optimization of multiple objectives, including the global utility, the personal utilities, the minimal personal utility, the resource utilization, the users' satisfaction degrees, etc. Auction-based allocations cannot achieve the same.

The design of the EGPM algorithm pays special attention to the three "evaluation indices": bandwidth utilization, demand satisfaction, and fairness. These are in fact rather common measures for network performance. The former two indices are embodied in Eq. (4). Proposition 5 for the equation implies that the EGPM algorithm will be able to narrow the gap between bandwidth resources and the demand and maximize the utilization of the links bandwidth. Max-min fairness is embodied in Eq. (3) and assured by Proposition 1.

\subsection{Paper structure}

Section 2 presents the model of bandwidth allocation problem. In Section 3, we give an overview of the EGPM architecture. In Sections 4 and 5, the evolution of the allocation policy and that of the pricing policy are addressed, respectively. The convergence of the EGPM algorithm is discussed and proved in Section 6. Section 7 presents the EGPM algorithm which is a synthesis of the results of the previous sections. In Section 8, we present the performance of EGPM-based on simulation, and compare it with GPM, genetic algorithm and other well-known bandwidth allocation approaches. Finally, we draw conclusions in Section 9. 


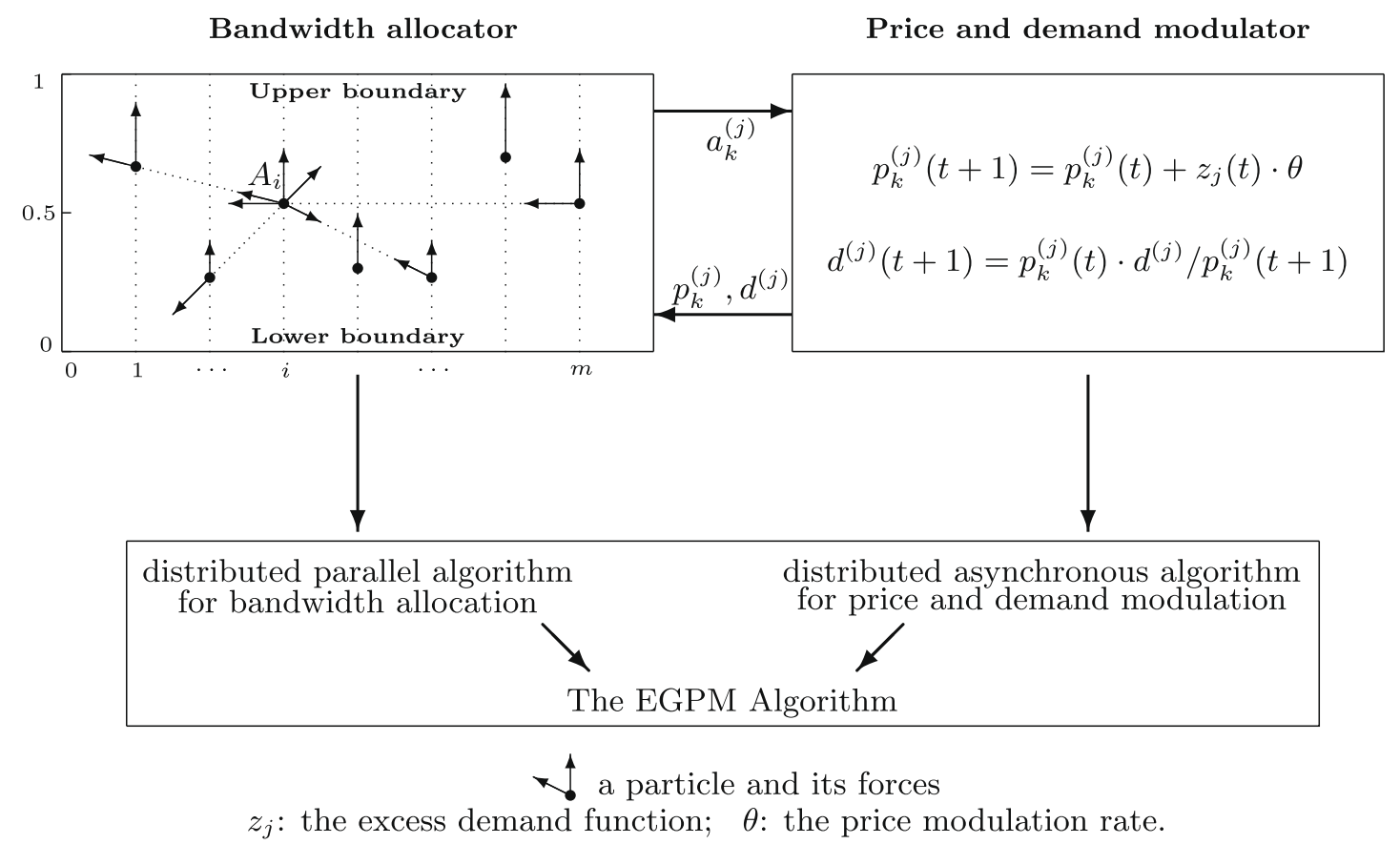

Fig. 1. The EGPM architecture.

\section{Problem model for bandwidth allocation}

The following are the important issues to consider in our solution.

1. Allocation effectiveness: the customers should be satisfied, the available bandwidths should be utilized as fully as possible, and the allocation should be fair.

2. Pricing strategy: how the various bandwidths should be priced in order to maximize every link's and network's revenue.

3. Parallel allocation: the allocation method should be easy to be implemented in a distributed fashion with minimal communications among the computing entities.

The first issue above presents specific objectives which are common to most bandwidth allocation problems and solutions. In our approach, the degree of this objective being met is measured by the demand satisfaction level (DSL), the bandwidth utilization level (BUL), and a fairness term. Every link's revenue and network's revenue are two artificial objectives in our EGPM algorithm, which are means to optimizing the real objectives of the problem. EGPM purports to address all the above issues.

In the bandwidth allocation problem, a source-destination pair communicate through a channel associated with the pair, and have a certain demand for bandwidth. The channel can be realized through one or more paths into which the channel's traffic would be split. We consider the scenario with a given number of links and their capacities, a given number of channels and their demands for bandwidth, and a given number of paths per each of these channels. This is the case of a static environment. Our approach applies also to the dynamic case where these parameters may change over time. If the environment does change during the calculation of an allocation, the EGPM algorithm will allocate the bandwidths based on the current calculation and then perform a new calculation from the beginning according to the new conditions.

The network is an undirected graph of $N$ nodes and $m$ links, and there are $J$ channels. The main parameters of any problem instance are as follows.

$A_{i}$ : The $i$ th physical link $(i=\overline{1, m})$.

$T^{(j)}$ : The $j$ th channel $(j=\overline{1, J} ; J \leqslant N \times(N-1) / 2)$.

$n_{j}$ : The number of the paths of channel

$T^{(j)}\left(n=\sum_{j=1}^{J} n_{j}\right)$.

$T_{k}^{(j)}$ : The $k$ th path of the $j$ th channel.

$r_{i}$ : The maximum bandwidth of link $A_{i}$.

$d^{(j)}$ : The median of the bandwidth demand of channel $T^{(j)}$.

$\varsigma_{j}$ : The mean square deviation of the bandwidth demand of channel $T^{(j)}$.

$x_{i k}^{(j)}: x_{i k}^{(j)}=1$ if path $T_{k}^{(j)}$ passes through $\operatorname{link} A_{i}$; otherwise 0 .

$a_{i k}^{(j)}$ : The bandwidth of link $A_{i}$ that is allotted to path $T_{k}^{(j)}$.

$p_{k}^{(j)}$ : The price per unit bandwidth that path $T_{k}^{(j)}$ has to pay.

$a_{k}^{(j)}$ : The actual bandwidth obtained by path $T_{k}^{(j)}$-that is,

$a_{k}^{(j)}=\min _{i}\left\{a_{i k}^{(j)} \mid \forall x_{i k}^{(j)}=1\right\} .^{2}$

Table 1 shows a snapshot of allocation.

The above parameters of the problem fall into several categories, as in Table 2.

The bandwidth allocation problem is defined as:

${ }^{2}$ If $x_{i k}^{(j)}=0, a_{i k}^{(j)}=0$. 
Table 1

The bandwidth allocation problem.

\begin{tabular}{|c|c|c|c|c|c|c|c|c|c|}
\hline \multirow[t]{2}{*}{ Link } & \multicolumn{9}{|l|}{ Path } \\
\hline & $T_{1}^{(1)}$ & $\cdots$ & $T_{n_{1}}^{(1)}$ & $\cdots$ & $T_{1}^{(j)}$ & $\cdots$ & $T_{n_{j}}^{(j)}$ & $\cdots$ & Link bandwidth \\
\hline$A_{1}$ & $x_{11}^{(1)}, a_{11}^{(1)}$ & $\cdots$ & $x_{1, n_{1}}^{(1)}, a_{1, n_{1}}^{(1)}$ & $\cdots$ & $x_{11}^{(j)}, a_{11}^{(j)}$ & $\cdots$ & $x_{1, n_{j}}^{(j)}, a_{1, n_{j}}^{(j)}$ & & $r_{1}$ \\
\hline$\vdots$ & $\vdots$ & & $\vdots$ & & $\vdots$ & & $\vdots$ & & $\vdots$ \\
\hline$A_{i}$ & $x_{i 1}^{(1)}, a_{i 1}^{(1)}$ & $\cdots$ & $x_{i, n_{1}}^{(1)}, a_{i, n_{1}}^{(1)}$ & $\cdots$ & $x_{i 1}^{(j)}, a_{i 1}^{(j)}$ & $\cdots$ & $x_{i, n_{j}}^{(j)}, a_{i, n_{j}}^{(j)}$ & $\cdots$ & $r_{i}$ \\
\hline$A_{m}$ & $x_{m 1}^{(1)}, a_{m 1}^{(1)}$ & $\cdots$ & $x_{m, n_{1}}^{(1)}, a_{m, n_{1}}^{(1)}$ & $\cdots$ & $x_{m 1}^{(j)}, a_{m 1}^{(j)}$ & $\cdots$ & $x_{m, n_{j}}^{(j)}, a_{m, n_{j}}^{(j)}$ & $\cdots$ & $r_{m}$ \\
\hline Allotted bandwidths (price) to paths & $p_{1}^{(1)}, a p_{1}^{(1)}$ & $\cdots$ & $p_{n_{1}}^{(1)}, a_{n_{1}}^{(1)}$ & $\cdots$ & $p_{1}^{(j)}, a_{1}^{(j)}$ & $\cdots$ & $p_{n_{j}}^{(j)}, a_{n_{j}}^{(j)}$ & $\cdots$ & \\
\hline Required bandwidths of channels & & $d^{(1)}, \varsigma$ & & $\cdots$ & & $d^{(j)}, \varsigma_{j}$ & & $\cdots$ & \\
\hline
\end{tabular}

Table 2

The notations.

\begin{tabular}{lll}
\hline Category & Parameter $(\mathrm{s})$ & During calculation \\
\hline The network & $A_{i}, T^{(j)}, T_{k}^{(j)}, N, m, J, n_{j}, x_{i k}^{(j)}$ & Fixed \\
Supply and demand & $r_{i}, d^{(j)}(t=0), \varsigma_{j}$ & Fixed \\
Solution & $a_{k}^{(j)}$ & Changing \\
Artificial variables & $d^{(j)}(t>0), a_{i k}^{(j)}, p_{k}^{(j)}$ & Changing \\
\hline
\end{tabular}

\section{Bandwidth allocation problem:}

Try to guarantee: satisfaction indicator (SI).

Maximize: demand satisfaction level (DSL), bandwidth utilization level (BUL), and fairness.

Given conditions: $N, m, J, n_{j}, r_{i}, d^{(j)}(t=0), \varsigma_{j}, x_{i k}^{(j)}$, and topology.

Solution: $a_{k}^{(j)}$.

Definition 1. The satisfaction indicator is a logical variable indicating whether the demand of a channel is met or not.

$\mathrm{SI}^{(j)}= \begin{cases}\text { Yes } & \sum_{k=1}^{n_{j}} a_{k}^{(j)}(t) \in\left[d^{(j)}-\varsigma_{j}, d^{(j)}+\varsigma_{j}\right] \\ \text { No } & \text { otherwise. }\end{cases}$

Definition 2. The demand satisfaction level of a channel (source-destination pair) shows the actual degree of satisfaction (or dissatisfaction) of the channel.

$\operatorname{DSL}^{(j)}=\frac{\sum_{k=1}^{n_{j}} a_{k}^{(j)}(t)}{d^{(j)}(t)} \times 100 \%$.

Definition 3. The bandwidth utilization level of every link is defined as

$\mathrm{BUL}_{i}=\frac{\sum_{j=1}^{p} \sum_{k=1}^{n_{j}} a_{k}^{(j)}(t) \cdot x_{i k}^{(j)}(t)}{r_{i}(t)} \times 100 \%$

Definition 4. The fairness of the allocation is measured by (according to [26])

$f\left(y_{1}, y_{2}, \ldots, y_{J}\right)=\left(\sum_{j=1}^{J} y_{j}\right)^{2} / J \sum_{j=1}^{J} y_{j}^{2}$, where $y_{1}, y_{2}, \ldots, y_{J}$ are the bandwidths allocated to $J$ channels, respectively.

In addition, we introduce two artificial measures-the network's revenue and every link's revenue-and some artificial variables $\left(d^{(j)}(t>0), a_{i k}^{(j)}, p_{k}^{(j)}\right)$ into the EGPM algorithm which are useful in the derivation of the final solution.

Definition 5. The revenue of link $A_{i}$ is given by

$\sum_{j=1}^{J} \sum_{k=1}^{n_{j}} p_{k}^{(j)}(t) \cdot a_{k}^{(j)}(t) \cdot x_{i k}^{(j)}(t)$

and the revenue of the network is the sum of the revenues of all the links.

The former two indices are embodied in Eq. (4). According to Proposition 5, the EGPM algorithm will always result in a decrease of Eq. (4)-i.e., the algorithm will narrow the gap between bandwidth resources and the demand, and will increase the utilization of the links' bandwidth. Max-min fair allocation can be obtained by the EGPM algorithm, which is assured by Proposition 6 as well as Proposition 1. Max-min fairness is embodied in Eq. (3). The degree of fairness achieved is quantified by the fairness measure $f($ ) defined above.

\section{The EGPM architecture}

As shown in Fig. 1, our EGPM-based approach and its corresponding algorithm are divided into two parts: (1) dynamic allocation of network bandwidth based on GPM, and (2) dynamic modulation of prices and demands for network bandwidth.

GPM uses a resource force-field to model the interactions of the network entities [1]. A similar force-field is used here by EGPM, which consists of numerous particles and forces. A particle with its own dynamic equations represents an entity of the network being modeled, a network link in this case; each force with its own time-varying properties represents a certain kind social interaction among the network entities. The evolution process is iterative: at every step the allocation module would produce an allocation based on which the modulator module would generate a new set of modulated prices and demands. The iteration terminates when an equilibrium is reached. In 
this paper, the two modules are combined in a single EGPM algorithm.

A particle in the resource force-field would move along a certain trajectory under the influence of a composite force. The particles' behaviors are characterized by the following.

- Each particle has an autonomous self-driving force corresponding to the "personality" of the network link being modeled.

- The stimulus of a particle is a function of the particle's objective, utility, and intention, which presents a multiple objective optimization problem to be solved.

- There are a variety of interactive forces among the particles, including unilateral forces, corresponding to the various kinds of social interaction that are possible in a network.

\section{Evolution of the allocation policy}

The allocation module accepts new parameter values at every step of the iterative process to produce an allocation which is closer to equilibrium than the previous allocation. Since the evolution of the allocation policy in GPM and that in EGPM are essentially the same, we only highlight the relevant definitions and properties here. For further details and the missing proofs, we refer the reader to [1].

The mathematical model involving $m$ links and $n$ paths is defined as follows. We refer to a particle in the forcefield that represents a link in the network a link particle.

Definition 6. Let $u_{i}(t)$ be the distance of link particle $A_{i}$ from the upper boundary of the force-field at time $t$, and let $J(t)$ be the total utility of all link particles. We define

$u_{i}(t)=\exp \left[-\sum_{j=1}^{J} \sum_{k=1}^{n_{j}} p_{k}^{(j)}(t) \cdot a_{k}^{(j)}(t) \cdot x_{i k}^{(j)}(t)\right]$,

$J(t)=\sum_{i=1}^{m} u_{i}(t)$

The larger the value of the double summation in Eq. (1) (hence the smaller the value of $u_{i}(t)$ ), the more profit the $i$ th link earns. The optimization problem here is posed as a minimization problem and so the term is negated. The exponential function makes $u_{i}(t)$ fall between 0 and 1 . $u_{i}(t)$ indeed is the utility of link particle $A_{i}$. The distance between the bottom and the upper boundary is set to be 1 . As for the total utility (Eq. (2)), the smaller the value of $J(t)$ the better.

Definition 7. At time $t$, the potential energy function $P(t)$, which is created by the upward gravitational force of the force-field, is defined by

$P(t)=\varepsilon^{2} \ln \sum_{i=1}^{m} \exp \left[-u_{i}^{2}(t) / 2 \varepsilon^{2}\right]$,

where $0<\varepsilon<1$ is a parameter to be tuned in the implementation, which affects directly the convergence speed.
The smaller the value of $P(t)$ the better. The decrease of this value implies the increase of the minimal utility among all the links (see Proposition 1). By this equation, we consider not only the aggregate, but also the individual personal utilities, in particular the minimum one, when trying to optimize the network bandwidth allocation. $\varepsilon$ represents the strength of the upward gravitational force in the force-field. The larger the value of $\varepsilon$ the faster the particles would move up; hence, $\varepsilon$ influences the convergence speed of the allocation process. Too fast a speed however might result in some of the possible solutions being skipped over, and so $\varepsilon$ needs to be carefully adjusted in order to maximize the users' satisfaction.

Definition 8. At time $t$, the potential interaction energy function, $Q(t)$, is defined by

$$
\begin{aligned}
Q(t)= & \beta_{1} \sum_{i=1}^{m}\left|\sum_{j=1}^{J} \sum_{k=1}^{n_{j}} a_{k}^{(j)}(t)-r_{i}(t)\right|^{2} \\
& +\beta_{2} \sum_{j=1}^{J}\left|\sum_{k=1}^{n_{j}} a_{k}^{(j)}(t)-d^{(j)}(t)\right|^{2},
\end{aligned}
$$

where $0<\beta_{1}, \beta_{2}<1$.

The smaller the value of $Q(t)$ the better. $\beta_{1}$ and $\beta_{2}$ are the weights assigned to the amount of available link bandwidth and the degree of satisfaction of the path demands, respectively.

Eq. (4) quantifies the effect of interactions among the links during bandwidth allocation. The first and the second term of $Q(t)$ can be seen as penalty functions due to the constraints on the utilization of resources (i.e., link bandwidth) and the satisfaction of the users (i.e., the channels), respectively. Therefore, resource utilization and users' satisfaction can be explicitly included as part of optimization objectives through some appropriate choices of the coefficients $\beta_{1}$ and $\beta_{2}$. There exist specific interactive forces among the particles, and these forces may cause the potential energy components represented by the first and second terms of $Q(t)$ to decrease.

Definition 9. The dynamics of particle $A_{i}$ are defined by

$\Delta a_{i k}^{(j)}(t)=-\lambda_{1} \frac{\partial u_{i}(t)}{\partial a_{i k}^{(j)}(t)}-\lambda_{2} \frac{\partial J(t)}{\partial a_{i k}^{(j)}(t)}-\lambda_{3} \frac{\partial P(t)}{\partial a_{i k}^{(j)}(t)}-\lambda_{4} \frac{\partial Q(t)}{\partial a_{i k}^{(j)}(t)}$,

where $\lambda_{1}, \lambda_{2}, \lambda_{3}$ are one-dimensional coefficient vectors, and $\lambda_{4}$ is a two-dimensional coefficient vector. $\lambda_{1}=$ $\left(\lambda_{i}^{(1)}\right)_{m \times 1}$, where $\lambda_{i}^{(1)}$ represents the strength of $A_{i}$ to pursue personal profit, $0 \leqslant \lambda_{i}^{(1)} \leqslant 1 . \lambda_{2}=\left(\lambda_{i}^{(2)}\right)_{m \times 1}$, where $\lambda_{i}^{(2)}$ represents the strength of $A_{i}$ to consider the aggregate profit of all the links, $0 \leqslant \lambda_{i}^{(2)} \leqslant 1 . \lambda_{3}=\left(\lambda_{i}^{(3)}\right)_{m \times 1}$, where $\lambda_{i}^{(3)}$ represents the strength of $A_{i}$ to increase the minimal personal profit among all the link particles, $0 \leqslant \lambda_{i}^{(3)} \leqslant 1$. $\lambda_{4}=\left(\lambda_{i u}^{(4)}\right)_{m \times m}$, where $\lambda_{i u}^{(4)}$ represents the strength of $A_{i}$ to interact with $A_{u}, 0 \leqslant \lambda_{i u}^{(4)} \leqslant 1$.

$A_{i}$ updates $a_{i k}^{(j)}$ according to Eq. (5)-that is, $a_{i k}^{(j)}(t+$ $1)=a_{i k}^{(j)}(t)+\Delta a_{i k}^{(j)}(t)$. We can therefore obtain the radial 
velocity of link particle $A_{i}$ along the vertical direction towards the upper boundary of gravitational field by the equation

$v_{i}=d u_{i} / d t=\sum_{j=1}^{J} \sum_{k=1}^{n_{j}} \frac{\partial u_{i}}{\partial a_{i k}^{(j)}} \frac{d a_{i k}^{(j)}}{d t}$.

Proposition 1. The decrease of the potential energy $P(t)$ of the gravitational field will result in the increase of the profit of the link whose profit is the minimum among all the links.

Proof. Let $U(t)=\max _{i} u_{i}^{2}(t)$. Because $U(t)=\max _{i} u_{i}^{2}(t) \leqslant$ $\sum_{i=1}^{m} u_{i}^{2}(t) \leqslant m \cdot \max _{i} u_{i}^{2}(t)=m \cdot U(t)$, we have $\left[e^{\frac{U(t)}{2 \varepsilon^{2}}}\right]^{2 \varepsilon^{2}} \leqslant$ $\left[\sum_{i=1}^{m} e^{\frac{u_{i}^{2}(t)}{2 \varepsilon^{2}}}\right]^{2 \varepsilon^{2}} \leqslant\left[m e^{\frac{U(t)}{2 \varepsilon^{2}}}\right]^{2 \varepsilon^{2}}$.

Simultaneously taking the logarithm of each side of the above equation leads to

$$
\begin{aligned}
& U(t) \leqslant 2 \varepsilon^{2} \ln \sum_{i=1}^{m} e^{\frac{u_{i}^{2}(t)}{2 \varepsilon^{2}}} \leqslant U(t)+2 \varepsilon^{2} \ln m, \\
& 2 \varepsilon^{2} \ln \sum_{i=1}^{m} e^{\frac{u_{i}^{2}(t)}{2 \varepsilon^{2}}} \geqslant U(t) \geqslant 2 \varepsilon^{2} \ln \sum_{i=1}^{m} e^{\frac{u_{i}^{2}(t)}{2 \varepsilon^{2}}}-2 \varepsilon^{2} \ln m, \\
& 2 P(t) \geqslant \max _{i} u_{i}(t) \geqslant 2 P(t)-2 \varepsilon^{2} \ln m .
\end{aligned}
$$

Since $m$ is the number of links, $2 \varepsilon^{2} \ln m$ is a constant. $P(t)$ therefore represents the maximum among all the $u_{i}(t)$ 's associated with $A_{i}$, namely, the minimum of the personal profits obtained by a link at time $t$. Hence, decreasing $P(t)$ implies the increase of the profit of the link whose profit is the minimum among all the links.

Definition 10. Max-min Fairness [27]. A feasible allocation of bandwidth $a$ is max-min fair if and only if an increase of any bandwidth within the domain of feasible allocations will be at the cost of a decrease of some already smaller bandwidth allocation. Formally, for any other feasible distribution $Y$, if $y_{i k}^{(j)}>a_{i k}^{(j)}$ then there must exist some $\left(i^{\prime}, k^{\prime}, j^{\prime}\right)$ such that $a_{i^{\prime} k^{\prime}}^{\left(j^{\prime}\right)} \leqslant a_{i k}^{(j)}$ and $y_{i^{\prime} k^{\prime}}^{\left(i^{\prime}\right)}<a_{i^{\prime} k^{\prime}}^{\left(i^{\prime}\right)}$.

Proposition 2. The behavior of $A_{i}$, by the third term of $\Delta a_{i k}^{(j)}$ (Eq. (5) ), will always bring about an increase of the minimum profit obtained by a link, and this increase is directly proportional to the coefficient vector $\lambda_{3}$.

Proposition 3. The behavior of $A_{i}$ caused by the first and third terms of $\Delta a_{i k}^{(j)}$ (Eq. (5) ) will always result in an increase of the personal profit of $A_{i}$, and this increase is proportional to the coefficient vectors $\lambda_{1}$ and $\lambda_{3}$.

Proposition 4. The behavior of $A_{i}$ caused by the second term of $\Delta a_{i k}^{(j)}$ (Eq. (5) ) will increase the global utility of the network, which is directly proportional to the coefficient vector $\lambda_{2}$.

Proposition 5. The behavior of $A_{i}$ caused by the fourth term of $\Delta a_{i k}^{(j)}$ (Eq. (5) ) will narrow the gap between bandwidth resources and the demand, and the strength is proportional to the coefficient vector $\lambda_{4}$.
Proposition 6 (Max-min fair allocation). Max-min fair allocation can be obtained by updating the allotted a by Eq. (5).

\section{Evolution of the pricing policy}

In this section, we present the evolutionary model that describes mathematically the dynamic modulation of prices and demands of network bandwidth. We introduce an important function, the excess demand function, which is a key element of the price modulation process.

\subsection{Excess demand function}

Definition 11. The price vector $p^{*}=p_{1}^{*(1)}, \ldots, p_{k}^{*(j)}, \ldots, p_{n_{J}}^{*(J)}$, a solution of the bandwidth allocation problem $a=a_{1}^{(1)}, \ldots, a_{k}^{(j)}, \ldots, a_{n_{j}}^{(J)}$ is in equilibrium if and only if $a$ is a feasible solution, and

$z_{j}\left(p^{*}\right)=\sum_{k=1}^{n_{j}} a_{k}^{(j)}-d^{(j)}=0$.

The mapping $z$ is called the excess demand function and it has the following properties:

P1: $z$ is single valued and continuous for all $p>0$.

P2: $z$ satisfies Walras' law: $p \cdot z(p)=0$ for all $p>0$.

P3: $z$ is homogeneous of degree zero: $z(\alpha p)=z(p)$ for all $\alpha>0$.

P4: There is a scalar $v<0$ such that $z_{j}(p)>v$ for all $j$ and $p>0$.

P5: $\lim _{p(t) \rightarrow p}\left[\max _{j, k \in J K_{p}} z_{j}(p(t))\right]=\infty$, when $p(t)>0, p \neq$ 0 and $J K_{p}=\left\{j, k: p_{k}^{(j)}=0\right\} \neq \emptyset$.

Homogeneity is an elementary property of the excess demand function because the channels' price vector stays the same when the budget constraints are multiplied by a positive constant. Walras' law and the continuity property result from the channels' minimization problems when the preferences are strictly concave and locally non-satiated. P4 means that all the component functions of $z$ are bounded from below on $\mathbb{R}_{+}^{n}$; this is because the net supply of bandwidth to the channels cannot exceed the channels' total demand. By P5, all the bandwidths of the paths are desirable in the sense that when some of these bandwidths become free of charge, there will exist at least some channels whose excess demand functions become infinitely large. When $z$ has the properties P1-P5, the economy has at least a ray of equilibrium prices. ${ }^{3}$

The absolute value of the excess demand function is inversely proportional to the demand satisfaction level (Definition 2). EGPM's pricing policy aims at maximizing the demand satisfaction levels of the channels, and therefore the iterative price modulation processes in EGPM have to satisfy the conditions that (1) the absolute value of the excess demand function will be minimized, and (2) the modulation processes will converge.

\footnotetext{
${ }^{3}$ See for example Chapter 17 of [28] for more about the properties of excess demand functions.
} 


\subsection{Iterative price modulation processes}

The dynamic modulation of price in the EGPM algorithm is based on the following. The price will rise when the supply of bandwidth is less than the demands of bandwidth $\left(z_{j}<0\right)$, whereas the price will fall when the supply of bandwidth is more than the demands of bandwidth $\left(z_{j}>0\right)$. The tendency to increase or decrease in price is directly proportional to $\left|z_{j}\right|$. In economics, such a dynamic modulation of price is known as the tatonnement process. The continuous time tatonnement process, introduced by Samuelson [29], is described by the differential equation

$\dot{p}(t)=z(p(t))$,

where $\dot{p}(t)$ is the time derivative of $p(t)$. This process is usually interpreted as an "auction" run by a fictitious agent, which sets the prices until an equilibrium is reached. It can be shown that under the following stability condition C1, the process of Eq. (8) is globally stable, i.e., it converges to an equilibrium for any positive initial prices.

C1: There exists $p^{*}>0$ that solves Eq. (7) and satisfies $p^{*} \cdot z(p)>0$ for all $p>0$ for which $z(p) \neq 0$.

The convergence condition $\mathrm{C} 1$ can be interpreted as the weak axiom of revealed preferences between the equilibrium $p^{*}$ and any disequilibrium price vector. The simplest discrete time alternative for the process of Eq. (8) is the fixed-point iteration

$p(t+1)=p(t)+z(p(t))$,

where $t$ is the iteration index corresponding to the time instants at which the prices are modulated. Analyzing Eq. (9) instead of Eq. (8) suits the bandwidth allocation problem better, for which the price modulation process proceeds over discrete time instants. Unfortunately, Eq. (9) is not suitable for solving Eq. (7), because some of the prices may become negative during the iteration, for which $z$ is usually not defined. One way to obtain non-negative prices is to update $p_{k}^{(j)}(t)$ as follows:

$p_{k}^{(j)}(t+1)=\max \left\{0, p_{k}^{(j)}(t)+\theta \cdot z_{j}\left(p_{k}^{(j)}(t)\right)\right\}$,

where $\theta$ is called the price modulation rate which is a positive constant. The convergence of this process restricts the choice of $\theta$ and $p(t=0)$. The drawback of the process of Eq. (10) is that due to P5 the excess demand function is not finite if some prices become zero. It can be easily seen that the discrete time process of Eq. (10) does not converge under the same assumptions as the continuous time process of Eq. (8). For example, the convergence of process of Eq. (10) depends on the choice of the parameter $\theta$. Moreover, normalized discrete time processes tend to exhibit chaotic behavior. We give an alternative discrete time process which converges under assumptions that are very close to those required for the process of Eq. (8) to converge. This process can be seen as a variation of the fixed-point iteration of Eq. (9) and it is defined by the following formula:

$p(t+1)=p(t)+\mu_{t} \cdot z(p(t))$,

where the parameter $\mu_{t}$ is updated as follows:
Step 1: A scalar $\gamma_{t}>0$ is chosen such that $p(t)+\gamma_{t}$. $z(p(t))>0$, and $\gamma_{t}=\gamma_{t-1}$ for $t \geqslant 1$ if $p(t)+\gamma_{t-1} \cdot z(p(t))>0$.

Step 2: $\mu_{t}=\min \left\{\gamma_{t}, M /\|z(p(t))\|\right\}$, where $M>0$.

The first step above is to guarantee that the new prices are positive. When $p(t)>0$, there is a positive number $\gamma_{t}$ such that $p(t)+\gamma_{t} \cdot z(p(t))>0$. It follows that when the initial prices are positive, i.e., $p(0)>0$, all the prices obtained during the process are positive as well. The second step guarantees that $\mu_{t} \cdot z(p(t))$ is bounded in the Euclidean norm $\|\cdot\|$. As a result, the magnitude of the change of the price vector is bounded, namely $\| p(t+1)-$ $p(t)\|=\| \mu_{t} \cdot z(p(t)) \| \leqslant M$, where $M$ is an arbitrarily chosen positive number. This property is needed in the convergence analysis of the process. Note that by these two steps, $\mu_{t}$ is updated only when it is necessary, to obtain positive prices or to bound the changes by $M$.

The process of Eq. (11) satisfies the law of demand in the sense that the price of bandwidth would rise with excess demands (of the channels), and the price would fall with excess supply. Moreover, the prices are adjusted in proportion to the excess demands in a fashion similar to that in the process of Eq. (8).

\section{Convergence analysis of EGPM}

\subsection{Convergence analysis}

We prove in this section that the process of Eq. (11) converges when $z$ has the properties P1-P5 and satisfies C1 as well as C2 which is stated below. In the condition $\mathrm{C} 2$, vector $p^{*}$ is the same equilibrium vector for which $\mathrm{C} 1$ holds, for $E_{\varsigma}=\left\{p \in \mathbb{R}_{+}^{n}:\|z(p)\|<\varsigma\right\}$.

C2: There are positive scalars $\varsigma$ and $\sigma$ such that $p^{*} \cdot z(p) \geqslant \sigma\|z(p)\|^{2}$ for all $p \in E_{\varsigma}$.

Let us examine the geometrical interpretation of the conditions $\mathrm{C} 1$ and $\mathrm{C} 2$. The condition $\mathrm{C} 1$ means that the hyperplane $\left\{a \in \mathbb{R}^{n}: p^{*} \cdot a=0\right\}$ supports the set $\left\{a \in \mathbb{R}^{n}\right.$ : $a=z(p), p>0\}$ (see Fig. 2). The condition C2 means that this set is at least located locally around the origin, inside a ball which has its center at the ray of solutions $\left\{p: p=\lambda p^{*}, \lambda>0\right\}$. Hence we can write $p^{*} \cdot z \geqslant \sigma\|z\|^{2}$ equivalently as $\left\|p^{*} /(2 \sigma)-z\right\| \leqslant\left\|p^{*} /(2 \sigma)\right\|$.

The way in which the parameter $\mu_{t}$ is updated guarantees that the norm of the scaled excess demand, $\mu_{t} z$, is bounded by the constant $M$. As a result, the scaled excess demand is for all $p>0$ inside a ball centered at the ray of solutions, as illustrated in Fig. 2, where $\sigma=1 / 2$ and $\lambda=1$.

Theorem 1. Let $z$ have the properties P1-P5 and satisfy the conditions $\mathrm{C} 1-\mathrm{C} 2$. Then the process of Eq. (11) converges to an equilibrium for any $p(0)>0$. If there is a unique ray of equilibria, then there exists an $N \geqslant 0$ such that the convergence is monotonical when $t \geqslant N$.

The monotonical convergence of the sequence $\{p(t)\}_{t}$ to $\widetilde{p}$ means that $\|p(t)-\widetilde{p}\| \rightarrow 0$, when $t \rightarrow \infty$, and if $p(t) \neq \widetilde{p}$, then $\|p(t+1)-\widetilde{p}\|<\|p(t)-\widetilde{p}\|$. The following lemmas are used in the proof of Theorem 1 which can be found 


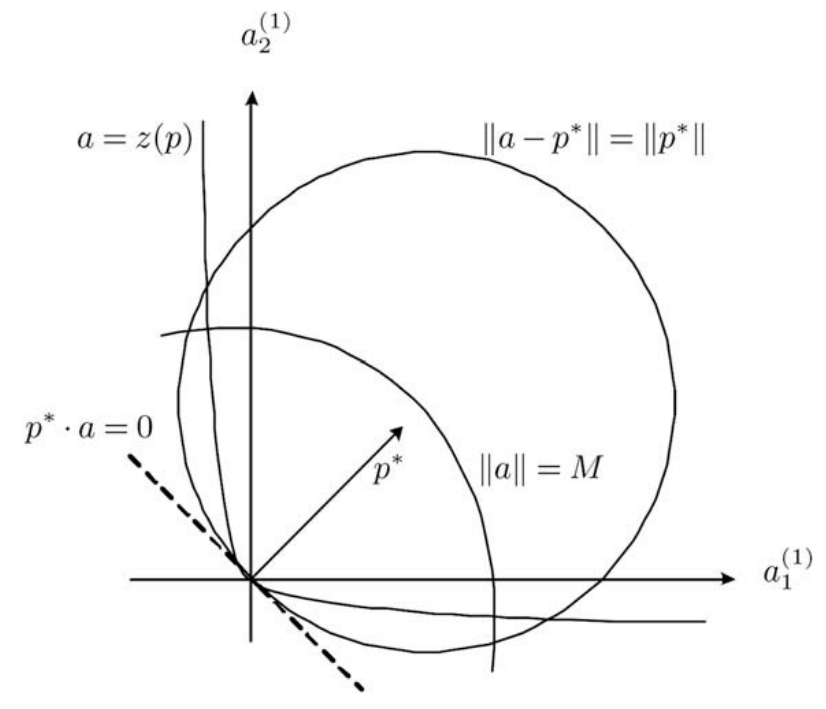

Fig. 2. Illustration of the convergence conditions.

in Appendix A. Here we let $B\left(p^{*}, \varsigma\right)$ denote the closed ball with radius $\varsigma>0$ and centered at $p^{*}$, i.e., $B\left(p^{*}, \varsigma\right)=$ $\left\{a \in \mathbb{R}^{n}:\left\|a-p^{*}\right\| \leqslant \varsigma\right\}$. The proofs of Lemmas $1-3$ are in Appendix A.

Lemma 1. Let the continuous mapping $z: B\left(p^{*}, r\right) \rightarrow \mathbb{R}^{n}$ satisfy Walras' law for all $p \in B\left(p^{*}, r\right)$, and let the inequality $p^{*} \cdot z(p) \geqslant\|z(p)\|^{2}$ hold for all $p \in B\left(p^{*}, r\right)$. If $p(0) \in B\left(p^{*}, r\right)$ and $\mu_{t} \leqslant 1$ for all $t$, then the iterative process $p(t+1)=$ $p(t)+\mu_{t} \cdot z(p(t))$ converges. When $0<\mu_{L} \leqslant \mu_{t}$, the process converges to the solution of $z(p)=0$.

Note that Lemma 1 does not assume that $p^{*}$ solves $z(p)=0$. It can be shown, however, that under the conditions of Lemma 1 vector $p^{*}$ is a solution. The following lemma shows that convergence is monotonical when there is a unique ray of equilibria.

Lemma 2. Let $z$ satisfy the same conditions as in Lemma 1 and let the iteration $p(t+1)=p(t)+\mu z(p(t)), \mu>0$, converge to a solution $\widetilde{p}$ for which there exists $\alpha>0$ such that

$\|z(p)\|^{2} \leqslant 2 \alpha z(p) \cdot \widetilde{p}$

for all $p \in B\left(p^{*}, r\right)$. Then the convergence is monotonical.

Lemma 3. If $z$ has the properties $\mathrm{P} 1, \mathrm{P} 3-\mathrm{P} 5$, and satisfies $\mathrm{C} 1-\mathrm{C} 2$, then there exists $\sigma>0$ such that $p^{*} \cdot \hat{z}(p) \geqslant$ $\sigma\|\hat{z}(p)\|^{2}$ for all $p>0$, where

$\hat{z}(p)= \begin{cases}M z(p) /\|z(p)\| & \text { if }\|z(p)\| \geqslant M \\ z(p) & \text { otherwise. }\end{cases}$

Theorem 2. When there is a unique equilibrium for the model of bandwidth allocation, the equilibrium prices solve the bandwidth optimization problem, i.e., the solution is globally Pareto optimal.

Proof. Assume that each path uses the process of Eq. (11) to update its price. The best price for a path is calculated from $\sum_{k=1}^{n_{j}} a_{k}^{(j)}=d^{(j)}$. At equilibrium, this is the condition that is satisfied. Furthermore, the links use Eq. (5) to calcu- late $a_{i k}^{(j)}$, which guarantees local optimality of the solution and the feasibility of the condition $\sum_{j=1}^{J} \sum_{k=1}^{n_{j}} a_{i k}^{(j)}(t) \leqslant$ $r_{i}(t), i=\overline{1, m}$. Uniqueness of the equilibrium guarantees that the locally feasible optimum solution is also the global optimum. Under these conditions, the outcome of the proposed approach is a solution to the bandwidth optimization problem.

Pareto optimality is a relevant criterion in a multiobjective problem setting. At Pareto optimum, we can find no other feasible solution that can improve some objectives without depriving other objectives. Theorem 2 states that, if the equilibrium is unique, then the outcome of the proposed approach is the optimal solution to the problem of maximizing individual revenues. If there are multiple equilibria, however, the resulting bandwidth allocation $a$ is only locally optimal.

From Theorem 2, if a solution $a$ is in equilibrium when the price vector is $p, a$ is an optimal solution, which implies that every link can obtain as large a profit as possible and so can the network for the total profit. As a result, individual optimization (which implies fairness) is consistent with global optimization and contributes to the efficiency of the latter.

Generally speaking, the utility function should satisfy the two conditions that (1) $u_{i}$ is a concave function, and (2) $u_{i}$ is monotonic.

In this paper, the utility function is defined by

$$
\begin{aligned}
& u_{i}(t)=\exp \left[-\sum_{j=1}^{J} \sum_{k=1}^{n_{j}} p_{k}^{(j)}(t) \cdot a_{k}^{(j)}(t) \cdot x_{i k}^{(j)}(t)\right], \\
& u_{i}^{\prime}(t)=-p_{k}^{(j)}(t) \cdot \exp \left[-\sum_{j=1}^{J} \sum_{k=1}^{n_{j}} p_{k}^{(j)}(t) \cdot a_{k}^{(j)}(t) \cdot x_{i k}^{(j)}(t)\right] \leqslant 0, \\
& u_{i}^{\prime \prime}(t)=\left[p_{k}^{(j)}(t)\right]^{2} \cdot \exp \left[-\sum_{j=1}^{J} \sum_{k=1}^{n_{j}} p_{k}^{(j)}(t) \cdot a_{k}^{(j)}(t) \cdot x_{i k}^{(j)}(t)\right] \geqslant 0 .
\end{aligned}
$$

When $p_{k}^{(j)}(t) \neq 0, u_{i}^{\prime}(t)<0$ and $u_{i}^{\prime \prime}(t)>0$. When $p_{k}^{(j)}(t) \neq$ $0, u_{i}$ is monotonic and concave.

The properties mentioned above have a deeper meaning. $u_{i}$ being a concave function implies that the improvement of the satisfaction level for bandwidth between two end nodes should be realized by means of balancing the bandwidth among several links. $u_{i}$ being monotonic implies that the more links there are and the more bandwidth each link has, the better.

\subsection{Convergence rate analysis}

Theorem 3. The iteration process of Eq. (11) converges to a solution $p^{*}$ in 2-rank.

Proof. Let $x_{t}$ denote $p(t)$; then $x_{t+1}$ denotes $p(t+1)$, and $x^{*}$ denotes $p^{*}$. Supposing that $x_{t+1}=f\left(x_{t}\right)$, the iteration process $p(t+1)=p(t)+\mu_{t} \cdot z(p(t))$ becomes

$f(x)=x+\mu_{t} \cdot z(x)$.

And we also have $f\left(x^{*}\right)=x^{*}+\mu_{t} \cdot z\left(x^{*}\right)$. Because $z\left(x^{*}\right)=0$, we have $f\left(x^{*}\right)=x^{*}$.

Since $z_{j}(p(t))=\sum_{k=1}^{n_{j}} a_{k}^{(j)}(t)-d^{(j)}(t)$, we have 


$$
\begin{aligned}
\mathbf{a}_{i k}^{(j)}(t+1)= & \mathbf{a}_{i k}^{(j)}(t)+\Delta \mathbf{a}_{i k}^{(j)}(t), \\
\Delta \mathbf{a}_{i k}^{(j)}(t)= & u_{i}\left(\lambda_{1} \mathbf{p}_{k}^{(j)}+\lambda_{3} w_{i}\right)-\lambda_{2}-2 \lambda_{4} \beta_{1}\left|\sum_{j=1}^{p} \sum_{k=1}^{n_{j}} \mathbf{a}_{k}^{(j)}-\mathbf{r}_{i}\right| \\
& -2 \lambda_{4} \beta_{2}\left|\sum_{k=1}^{n_{j}} \min _{i} \mathbf{a}_{i k}^{(j)}-\mathbf{d}^{(j)}\right| .
\end{aligned}
$$

We can obtain $z^{\prime}\left(x^{*}\right)=A \neq 1$ and $A \neq 0 . z(x)$ is continuously differentiable for two times. From the Taylor's equation, when $x$ sufficiently approaches $x^{*}$, we get

$z(x)=z\left(x^{*}\right)+A\left(x-x^{*}\right)+0.5 z^{\prime \prime}(\xi)\left(x-x^{*}\right)^{2}$.

Let $x^{*}=0$, we obtain

$z(x)=A x+O\left(x^{2}\right)$.

From Eq. (13) and $A \neq 1$, we get

$f(x)=x+\mu_{t} \cdot\left[A x+O\left(x^{2}\right)\right]=x+A \mu_{t} \cdot x+O\left(x^{2}\right)$.

Therefore

$\lim _{t \rightarrow \infty} \frac{x_{t+1}-x^{*}}{\left(x_{t}-x^{*}\right)^{n}}=c \Rightarrow \lim _{t \rightarrow \infty} \frac{x+A \mu_{t} \cdot x+O\left(x^{2}\right)-x^{*}}{\left(x-x^{*}\right)^{n}}=c \Rightarrow n=2$.

So the iteration process of Eq. (11) converges to a solution $p^{*}$ in 2-rank.

\section{The EGPM algorithm}

Here we present the EGPM algorithm which consists of a component for distributed parallel bandwidth allocation and a component for distributed asynchronous price-demands modulation.

\section{Input:}

$$
r_{i}, \varsigma_{j}, d^{(j)}, x_{i k}^{(j)}
$$

\section{Initialization:}

$t \leftarrow 0$

$\Delta t, \theta, \varepsilon, \beta_{1}, \beta_{2}, a_{i k}^{(j)}(t), p_{k}^{(j)}(t)$

2. $a_{k}^{(j)}(t) \leftarrow \min _{i} a_{i k}^{(j)}(t)$

$z_{j} \leftarrow \sum_{k=1}^{n_{j}} a_{k}^{(j)}(t)-d^{(j)}(t)$

Compute $v_{i}(t)$ according to Eq. (6)

3. while $\left(\left|z_{j}\right|>\varsigma_{j}\right.$ and $\left.v_{i}(t) \neq 0\right)$ do

(3.1) $p_{k}^{(j)}(t+\Delta t) \leftarrow p_{k}^{(j)}(t)+z_{j}(t) \cdot \theta$

$$
\text { if }\left(p_{k}^{(j)}(t+\Delta t) \leqslant 0\right)
$$$$
\theta \leftarrow \theta / 2
$$$$
p_{k}^{(j)}(t+\Delta t) \leftarrow p_{k}^{(j)}(t)+z_{j}(t) \cdot \theta
$$

$d^{(j)}(t+\Delta t) \leftarrow p_{k}^{(j)}(t) \cdot d^{(j)}(t) / p_{k}^{(j)}(t+\Delta t)$

$t \leftarrow t+\Delta t$

(3.2) Compute $u_{i}(t)$ according to Eq. (1)

Compute $v_{i}(t)$ according to Eq. (6)

Compute $d a_{i k}^{(j)}(t) / d t$ according to Eq. (5)

$a_{i k}^{(j)}(t) \leftarrow a_{i k}^{(j)}(t-\Delta t)+d a_{i k}^{(j)}(t) / d t$

if $\left(\sum_{j=1}^{l} \sum_{k=1}^{h} a_{i k}^{(j)}(t) \leqslant r_{i}\right.$ and $\left.\sum_{j=1}^{l} \sum_{k=1}^{h+1} a_{i k}^{(j)}(t)>r_{i}\right)$
$a_{i k}^{(j)}(t)=0$

$a_{k}^{(j)}(t) \leftarrow \min _{i} a_{i k}^{(j)}(t)$

$$
z_{j} \leftarrow \sum_{k=1}^{n_{j}} a_{k}^{(j)}(t)-d^{(j)}(t)
$$

(3.3) if $\left(\left|z_{j}(t)\right| \geqslant\left|z_{j}(t-\Delta t)\right|\right)$

$\theta \leftarrow \theta / 2$

$p_{k}^{(j)}(t) \leftarrow p_{k}^{(j)}(t-\Delta t)$

$d^{(j)}(t) \leftarrow d^{(j)}(t-\Delta t)$ $\varepsilon$ is a parameter to be tuned in the implementation, which affects directly the rate of convergence. $\theta$ is the price modulation rate. $\varsigma_{j}$ is the desired threshold of $z_{j}$, which should be input as $\sigma^{2}$ (variance) of $d^{(j)}$, and $d^{(j)}$ should be input as $\mu$ (mean) of $d^{(j)}$. Substeps 3.1 and 3.3 together implement the price and demands modulation, and Substep 3.2 implements the bandwidth allocation policy.

The process of network bandwidth allocation comprises real-time variation of the supply and demands of bandwidth. In the network, links are appended to or deleted from, the bandwidth demands between two end nodes arise and end, and the bandwidth supply or demands increase or decrease. The time quantum $\Delta t$ used by the iterative process is adjusted and modulated based on the speed of change of the supply and demands of bandwidth. $\Delta t$ should be made smaller when the supply and demands vary rapidly, and larger when the supply and demands vary tardily.

The $(a)_{m \times n}$ and $(p)_{1 \times n}$ can be initialized as: (1) some average values between 0 and 1 , or (2) random numbers between 0 and 1 . In fact, based on the experiments we have done, we found that the final results are not affected by how these variables are initialized. $\beta_{1}$ and $\beta_{2}$, the weights used in Eq. (4), can be initialized as any constants between 0 and 1 .

In Substep 3.1, when $p_{k}^{(j)}(t+\Delta t) \leqslant 0$ and $p_{k}^{(j)}(t)>0$, we perform " $\theta \leftarrow \theta / 2$ " one or more times to make $p_{k}^{(j)}(t+$ $\Delta t)>0$. In Substep 3.3, if $\left|z_{j}(t)\right| \geqslant\left|z_{j}(t-\Delta t)\right|$, which means the result of the $t$ th iteration is not better than the last iteration, we perform " $\theta \leftarrow \theta / 2$ ". It is possible to leap over better convergence points because $\theta$ is too big and convergence speed is too fast. By making $\theta$ smaller (a half), we can avoid this. In Substep 3.2, if $\left(\sum_{j=1}^{l} \sum_{k=1}^{h} a_{i k}^{(j)}(t) \leqslant\right.$ $r_{i}$ and $\left.\sum_{j=1}^{l} \sum_{k=1}^{h+1} a_{i k}^{(j)}(t)>r_{i}\right)$, we let all $a_{i k}^{(j)}(t)=0$ in order to make all $a_{i k}^{(j)}$ of the $i$ th row of the allocation matrix to be not larger than $r_{i}$.

Since the "actual bandwidth" obtained by a path is the smallest $a_{i k}^{(j)}$ over all the links that make up the path, we let $a_{k}^{(j)}(t) \leftarrow \min _{i} a_{i k}^{(j)}(t)$ in Substep 3.2. The individual $a_{i k}^{(j)}$,s are often larger than $a_{k}^{(j)}$. The wastage due to over-allocation however will not be present in the final solution as the $a_{i k}^{(j)}$ 's exist only in the computing process.

The EGPM algorithm can be executed to allocate network bandwidth in parallel, by dynamically modulating price and demands of network bandwidth in an asynchronous fashion. Although the solution at equilibrium as worked out by this asynchronous modulation of price and demands is a local equilibrium solution within a small time frame, it will approach the globally optimal solution within a large time frame. What is more, the asynchronous nature of the algorithm makes the algorithm adaptable to complex and fast changing network environments.

\section{Simulations and comparisons}

\subsection{Bandwidth allocation by EGPM}

\subsubsection{The parameters}

The main parameters of the EGPM algorithm are: $\varepsilon, \beta_{1}, \beta_{2}, \theta, m, n, J, \lambda_{1}=\left(\lambda_{i}^{(1)}\right)_{m \times 1}, \quad \lambda_{2}=\left(\lambda_{i}^{(2)}\right)_{m \times 1}, \quad \lambda_{3}=$ 
$\left(\lambda_{i}^{(3)}\right)_{m \times 1}, \quad \lambda_{4}=\left(\lambda_{i u}^{(4)}\right)_{m \times m}$ influence the convergence speeds of bandwidth allocation and price modulation, respectively. $\beta_{1}, \beta_{2}$ are the weights assigned to the available bandwidth and the satisfaction degree of the channels, respectively. $m, n, J$ are fixed given values reflecting the dimension of the problem.

Fig. 3 shows the evolutionary trajectories of 4 of 38 link particles in the force-field during problem solving. The simulation uses the following parameter values: $\varepsilon=$ $0.8, \beta_{1}=0.1, \beta_{2}=0.1, \theta=0.5, m=38, n=32, J=10$, $\lambda_{1}=\left(\lambda_{i}^{(1)}\right)_{m \times 1}, \quad \lambda_{2}=\left(\lambda_{i}^{(2)}\right)_{m \times 1}, \quad \lambda_{3}=\left(\lambda_{i}^{(3)}\right)_{m \times 1}, \lambda_{4}=\left(\lambda_{i u}^{(4)}\right)_{m \times m}$, and $\lambda_{i}^{(1)}, \lambda_{i}^{(2)}, \lambda_{i}^{(3)}, \lambda_{i u}^{(4)}$ are chosen at random from $[0,1]$. The relatively fast speed of convergence can be observed in Fig. 3. The $y$-axis is the dynamic state, $u_{i}$, of a particle, as defined by Eq. (1).

In Fig. 3, there is a huge dive in the trajectory of Particle 25. Modulated prices and demands may make bandwidth allocation of several links huge change at next iteration. When allocated bandwidth $a_{k}^{(j)}$ and prices $p_{k}^{(j)}$ are updated, dynamic state $u_{i}$ of link particles will change according to Eq. (1).

In addition, in Fig. 3, there is a decrease trend in the trajectory of Particle 33. That is, the dynamic state (representing link's revenue) of most link particles increases while that of a few of link particles decreases. Every link's revenue and network's revenue are two artificial objectives in our EGPM algorithm, which are means to optimizing the real objectives (SI, DSL, BUL, $f$ ) of the problem. Every link's revenue and network's revenue are all given attention to by EGPM. The dynamic state $u_{i}$ represents every link's revenue. When a link's revenue conflicts with network's revenue, the link will give in and the link's revenue will decrease.

$\lambda_{1}, \lambda_{2}, \lambda_{3}$, and $\lambda_{4}$ can be adjusted to suit the problem's objectives. $\lambda_{1}$ and $\lambda_{3}$ control the links' autonomy and selfinterest; $\lambda_{2}$ reflects the links' interest to improve the global state. $\lambda_{4}$ reflects the strength of the interaction among the links. For instance, when $\lambda_{1}=0, \lambda_{3}=0$ and $\lambda_{2}=1$, the links care for global profits only; when $\lambda_{1}=1, \lambda_{3}=1$ and $\lambda_{2}=0$, they care only for their own profits; and when $0 \leqslant \lambda_{1}, \lambda_{3}, \lambda_{2} \leqslant 1$, they opt for a balance.

Table 3 shows how different choices of $\lambda$ values influence the performance of EGPM. The numbers in the table are the average of two indices-bandwidth utilization (BUL) and the channels' satisfaction level (DSL). The simulation comprises more than 100 problems. We can see that when the link particles care for both global profits and their own profits, the performance of EGPM is the best.

\subsubsection{Effectiveness}

To see how EGPM reacts to the varying level of available bandwidth, we tested it using the bandwidth allocation problem instance in [14]. The network has 7 nodes, 11 links, 42 source-destination pairs (channels) and 84 virtual paths (two paths per channel), whose topology is shown in Fig. 4. In this problem, we set $n_{j}=2$ (for $j=\overline{1,42}$ ), the same as in [14]. Table 4 shows the channels and their bandwidth demands.

For the links' capacity $\left(r_{i}\right)$, two types of cases are considered: limited capacity $\left(r_{i}=240 \mathrm{Mbits} / \mathrm{s}\right.$ and $r_{i}=480 \mathrm{Mbits} /$ $\mathrm{s})$, and abundant capacity $\left(r_{i}=720 \mathrm{Mbits} / \mathrm{s}\right.$ and $r_{i}=960 \mathrm{M}-$ bits/s). We also include a "heterogeneous" case where link

Table 3

Allocation performance from different degrees of link selfishness.

\begin{tabular}{llll}
\hline The links' disposition & $\begin{array}{l}\text { Supply }> \\
\text { demand }\end{array}$ & $\begin{array}{l}\text { Supply } \approx \\
\text { demand }\end{array}$ & $\begin{array}{l}\text { Supply }< \\
\text { demand }\end{array}$ \\
\hline Initial allocation & 0.2 & 0.5 & 0.4 \\
Global profits only & 0.3 & 0.5 & 0.6 \\
Own profits only & 0.67 & 0.66 & 0.62 \\
Global profits as & 0.76 & 0.72 & 0.8 \\
well as own profits & & & \\
\hline
\end{tabular}

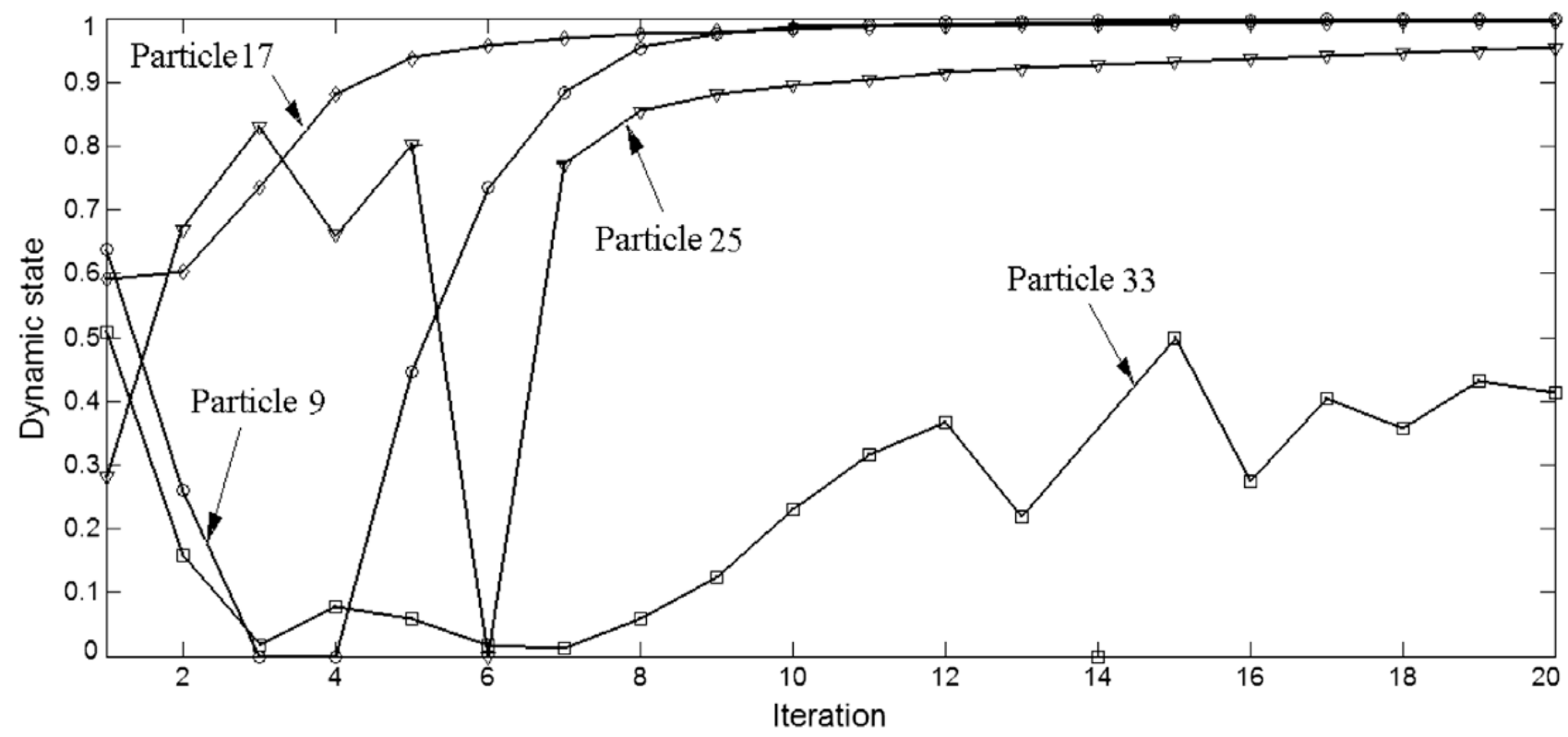

Fig. 3. The kinematics and dynamic trajectories of four selected particles in the force-field during bandwidth allocation. 


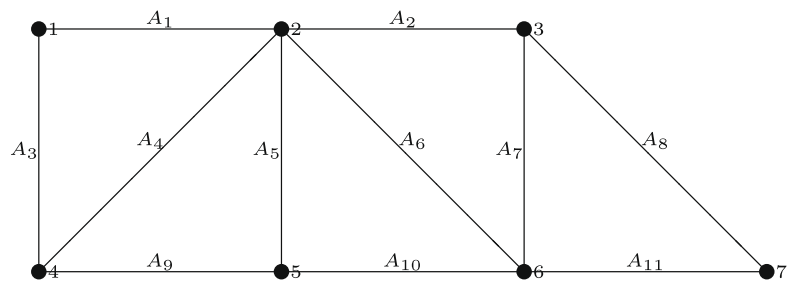

Fig. 4. A 7-node network.

capacity differs from link to link. The results are summarized in Table 5. From the table, we observe the following.

- The demands of all source-destination pairs (channels) are satisfied (according to SI, the satisfaction indicator) in all cases except the case of extreme scarce capacity ( $240 \mathrm{Mbits} / \mathrm{s}$ ) of which $75 \%$ of the demands are satisfied.

- The average BUL, average DSL, and fairness are reasonably high in all five cases, especially in the cases of scarce capacity.

- When the link capacity is plentiful, the satisfaction level (DSL) of all source-destination pairs (channels) can reach $100 \%$.

- When network capacity decreases rapidly, such as from 960 to $480 \mathrm{Mbits} / \mathrm{s}$, the performance indices do not seem to follow suit.

- Whether the network is homogeneous or heterogeneous in terms of link capacities is not a key factor for SI, BUL, DSL and fairness.

\subsubsection{Efficiency and parallelism}

In this section, we show the actual times used to compute the solutions, on a cluster. The machines of the cluster each has a Pentium $42.0 \mathrm{GHz}$ CPU with 512 Kbytes of L2 cache and 512 Mbytes of DDR SDRAM, and they are interconnected via Fast Ethernet.

The network of the problem is such that if there are $N$ nodes in the network, the number of source-destination pairs (channels) is $N \times(N-1)$. We use the number of nodes and channels to represent the "scale" of the problem. Convergence times and iterations for different scales using EGPM are shown in Table 6. The data come from using 1,4 , and 8 computing nodes of the cluster. "Increase" represents the percent of increase of the current scale from the previous scale.

As shown in Fig. 5, the convergence time of the sequential version increases exponentially with the scale, which is similar to all other exact methods. When parallelized, the convergence time drops significantly across the larger scale problems. The times for the smaller scale problems are dominated by the message exchange times, and the sequential version appears to be more efficient in that range. The number of iterations for all the cases, however, is more or less the same for either the sequential or the parallel versions.

The EGPM algorithm has the ability to carry out the allocation in a distributed manner with little communication overheads. The bandwidth allocation variables, $a_{i k}^{(j)}$ (see Table 1), can be computed and updated in parallel without any information exchange, which is the founda-

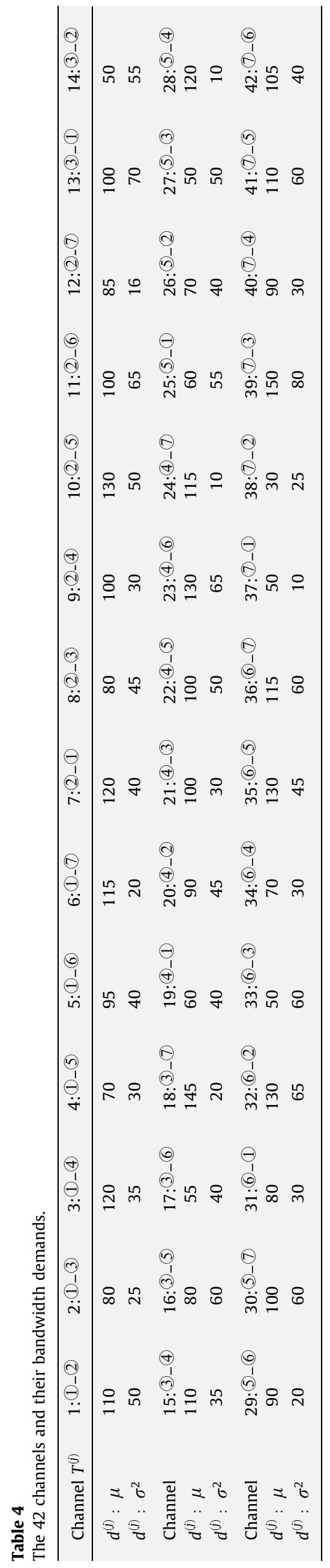


Table 5

Allocation results for different link capacities.

\begin{tabular}{|c|c|c|c|c|}
\hline & $(\mathrm{SI}=\mathrm{Yes}) / \mathrm{J}$ & Average BUL & Fairness f & Average DSL \\
\hline$r_{i}=240$ Mbits $/ \mathrm{s}$ (extremely scarce) & $32 / 42$ & 0.9134 & 0.4304 & 0.3619 \\
\hline$r_{i}=480 \mathrm{Mbits} / \mathrm{s}$ (scarce) & $42 / 42$ & 0.9646 & 0.7385 & 0.7449 \\
\hline$r_{i}=720 \mathrm{Mbits} / \mathrm{s}$ (plentiful) & $42 / 42$ & 0.9084 & 0.8601 & 1.0000 \\
\hline$r_{i}=960 \mathrm{Mbits} / \mathrm{s}$ (extremely plentiful) & $42 / 42$ & 0.7340 & 0.8972 & 1.0000 \\
\hline $\begin{array}{l}r_{i}=[560,480,320,480,560,480,400,480,560,480,480](\text { Mbits/s) } \\
\text { (Heterogeneous) }\end{array}$ & $42 / 42$ & 0.8036 & 0.7555 & 0.5233 \\
\hline
\end{tabular}

Table 6

Convergence times and iterations of EGPM.

\begin{tabular}{|c|c|c|c|c|c|c|c|c|c|c|c|c|c|}
\hline \multicolumn{2}{|l|}{ Scale } & \multicolumn{4}{|l|}{ Sequential } & \multicolumn{4}{|c|}{4 parallel nodes } & \multicolumn{4}{|c|}{8 parallel nodes } \\
\hline Nodes & Channels & Iterations & $\begin{array}{l}\text { Increase } \\
(\%)\end{array}$ & $\begin{array}{l}\text { Time } \\
\text { (s) }\end{array}$ & $\begin{array}{l}\text { Increase } \\
(\%)\end{array}$ & Iterations & $\begin{array}{l}\text { Increase } \\
(\%)\end{array}$ & $\begin{array}{l}\text { Time } \\
\text { (s) }\end{array}$ & $\begin{array}{l}\text { Increase } \\
(\%)\end{array}$ & Iterations & $\begin{array}{l}\text { Increase } \\
(\%)\end{array}$ & Time & Increase \\
\hline 4 & 12 & 103 & & 0.008 & & 134 & & 0.175 & & 112 & & 0.301 & \\
\hline 5 & 20 & 112 & 8.7 & 0.018 & 125.0 & 150 & 11.9 & 0.2 & 14.3 & 135 & 20.5 & 0.429 & 42.5 \\
\hline 6 & 30 & 130 & 16.1 & 0.029 & 61.1 & 171 & 14.0 & 0.245 & 22.5 & 164 & 21.5 & 0.5 & 16.6 \\
\hline 7 & 42 & 172 & 32.3 & 0.076 & 162.1 & 221 & 29.2 & 0.363 & 48.2 & 205 & 25.0 & 0.69 & 38.0 \\
\hline 8 & 56 & 230 & 33.7 & 0.141 & 85.5 & 297 & 34.4 & 0.386 & 6.3 & 304 & 48.3 & 0.87 & 26.1 \\
\hline 9 & 72 & 363 & 57.8 & 0.317 & 124.8 & 446 & 50.2 & 0.551 & 42.7 & 397 & 30.6 & 1.043 & 19.9 \\
\hline 10 & 90 & 544 & 49.9 & 0.69 & 117.7 & 563 & 26.2 & 0.843 & 53.0 & 622 & 56.7 & 1.564 & 50.0 \\
\hline 11 & 110 & 734 & 34.9 & 1.235 & 79.0 & 797 & 41.6 & 1.324 & 57.1 & 796 & 28.0 & 2.374 & 51.8 \\
\hline 12 & 132 & 979 & 33.4 & 2.101 & 70.1 & 968 & 21.5 & 1.611 & 21.7 & 1029 & 29.3 & 2.571 & 8.3 \\
\hline 13 & 156 & 1358 & 38.7 & 3.782 & 80.0 & 1360 & 40.5 & 2.51 & 55.8 & 1370 & 33.1 & 3.847 & 49.6 \\
\hline 14 & 182 & 1618 & 19.1 & 5.616 & 48.5 & 1934 & 42.2 & 4.21 & 67.7 & 1857 & 35.5 & 5.327 & 38.5 \\
\hline 15 & 210 & 2260 & 39.7 & 9.821 & 74.9 & 2213 & 14.4 & 5.11 & 21.4 & 2724 & 46.7 & 7.708 & 44.7 \\
\hline 16 & 240 & 2865 & 26.8 & 15.12 & 54.0 & 3221 & 45.5 & 8.58 & 67.9 & 2806 & 3.0 & 6.567 & -14.8 \\
\hline 17 & 272 & 3627 & 26.6 & 23.578 & 55.9 & 4159 & 29.1 & 13.85 & 61.4 & 3928 & 40.0 & 12.801 & 94.9 \\
\hline 18 & 306 & 4941 & 36.2 & 37.534 & 59.2 & 4885 & 17.5 & 17.37 & 25.4 & 4298 & 9.4 & 13.898 & 8.6 \\
\hline 19 & 342 & 5442 & 10.1 & 50.188 & 33.7 & 5342 & 9.4 & 21.17 & 21.9 & 5328 & 24.0 & 18.618 & 34.0 \\
\hline 20 & 380 & 7528 & 38.3 & 78.089 & 55.6 & 7547 & 41.3 & 31.913 & 50.7 & 6897 & 29.4 & 25.627 & 37.6 \\
\hline
\end{tabular}

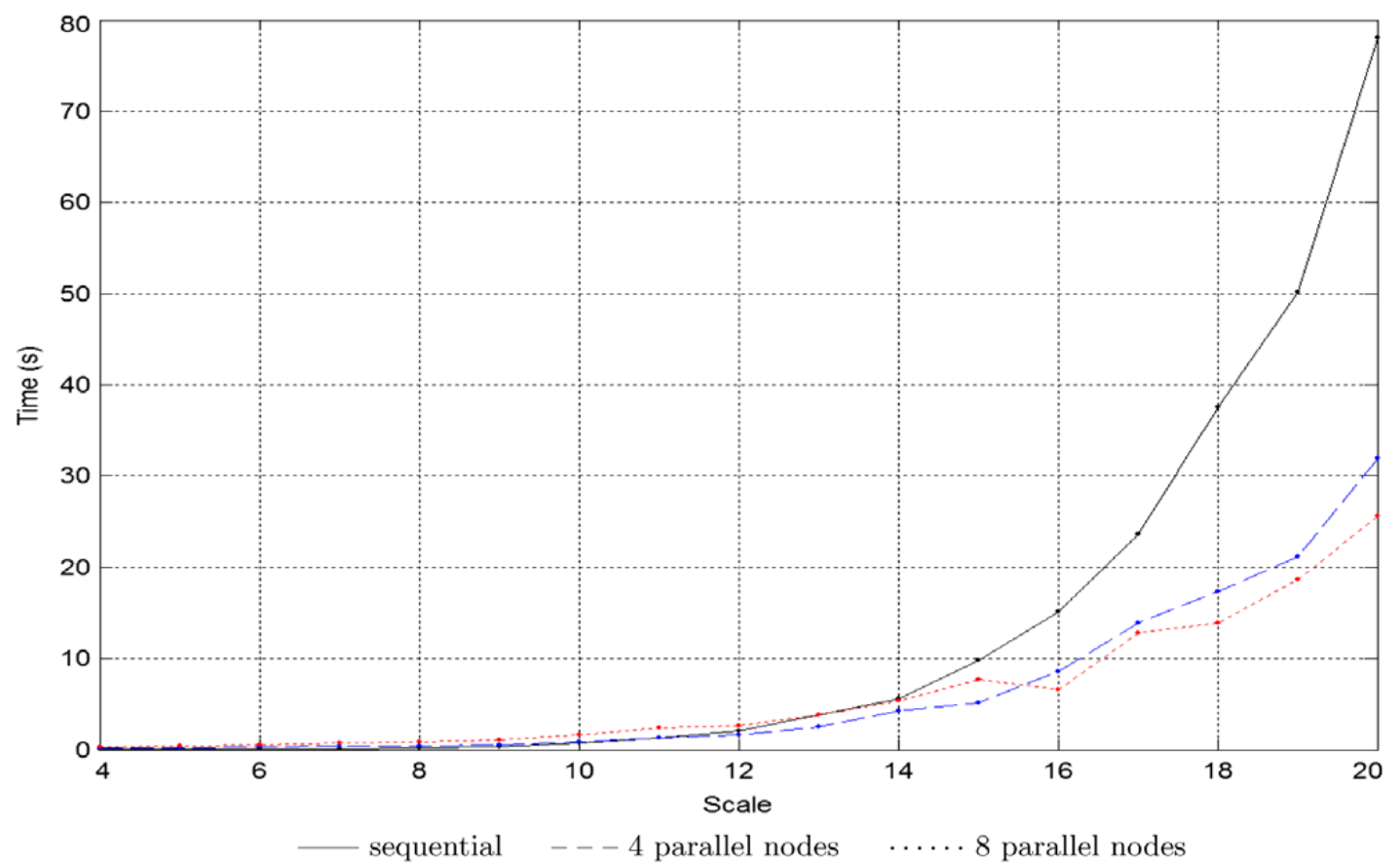

Fig. 5. Convergence times of EGPM using one or more processors.

tion of EGPM's parallelism. There is some information exchange between entries in a column (in Table 1) to com- pute and update the actual bandwidth allocation to the paths $\left(a_{k}^{(j)}\right)$, the price $\left(p_{k}^{(j)}\right)$ and the demand variable 
$\left(d^{(j)}(t>0)\right)$; as well as some information exchange between entries in a row in order to satisfy the links' capacity restrictions. These exchanges of information in a row or column are responsible for the less than ideal speedup that can be achieved using parallel implementation.

\subsection{Comparison with GPM}

Fig. 6 shows an example highlighting the difference between demand and allocation in a dynamic environment where the bandwidth demands may change with time. In Fig. 6a, the curved line corresponds to the demand and the rectangles the supply (allocated bandwidth) over a period of time. $T$ in the figure denotes the length of a run of the GPM algorithm to calculate the equilibrium allocation. Because the allocation will not change until the equilibrium is reached in a run of the GPM algorithm, the allocation stays unchanged between any two consecutive runs in the figure.

The EGPM algorithm allows links to trade resources to settle excess demand at every iteration. In Fig. 6b, the two curved lines represent the change of the demand and the allocated bandwidth, respectively. For both GPM and EGPM, the shaded regions represent the "excess de- mand function" (i.e., over- or under-allocation). The shaded regions of both GPM and EGPM shrink in size when the interval of environmental changes becomes longer. When that interval is small (i.e., the changes are frequent) the excess demand function of EGPM is smaller and EGPM is preferable. However, because EGPM needs to renew the allocation at every iteration step in a run before reaching equilibrium, it takes a longer time than GPM. Hence, when the interval of change is excessively long, GPM might have an advantage.

Next, we compare the satisfaction level. There is a direct relationship between the satisfaction level and the excess demand function: when the level is low, the excess demand function is large and the allocation is not preferable, and if the level is high, the excess demand function is small and the allocation is well done. The level reaches 1 if and when the allocation matches exactly the demand.

In a static environment where the demands of the paths do not change, the satisfaction level changes in a fashion which is as shown in Fig. 7a. The figure shows that the GPM algorithm takes shorter time $(T)$ to complete its run than the iterative EGPM algorithm. It can be easily seen that, for a static environment, if $T$ is small, GPM is preferable, and if $T$ is large, EGPM is preferable.

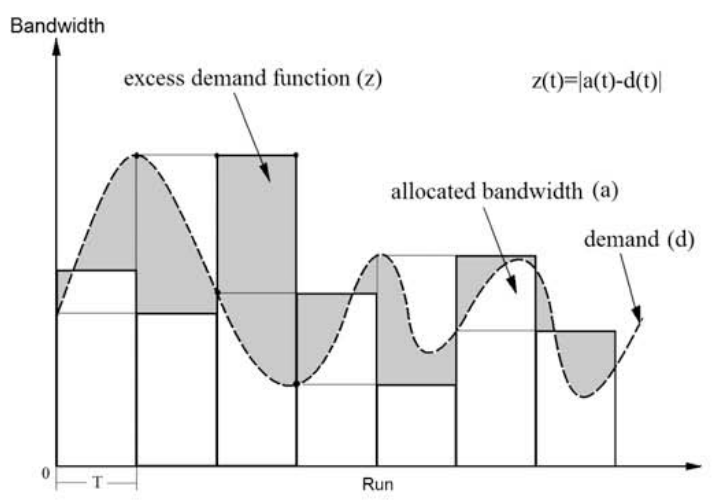

(a) GPM

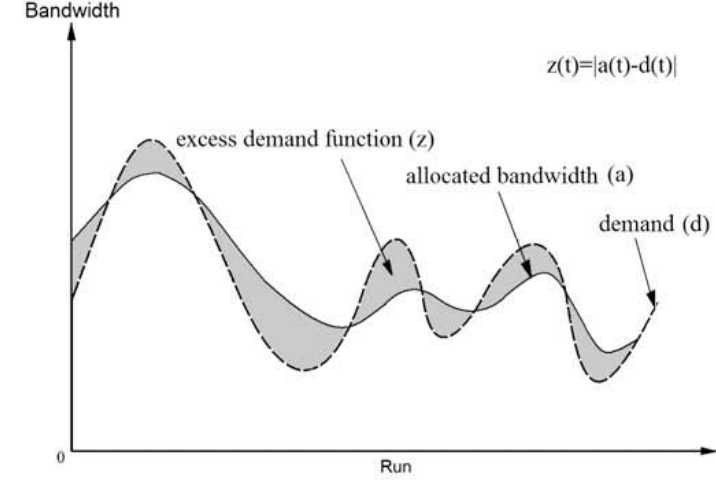

(b) EGPM

Fig. 6. Excess demands in a dynamic environment.

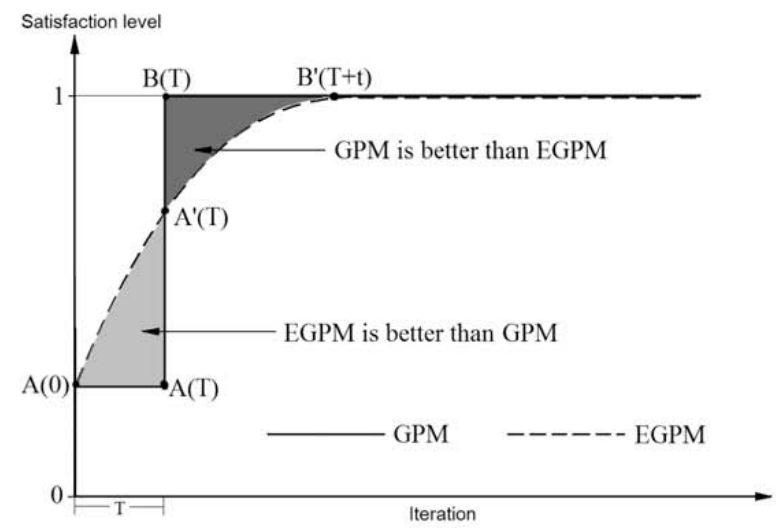

(a) static environment

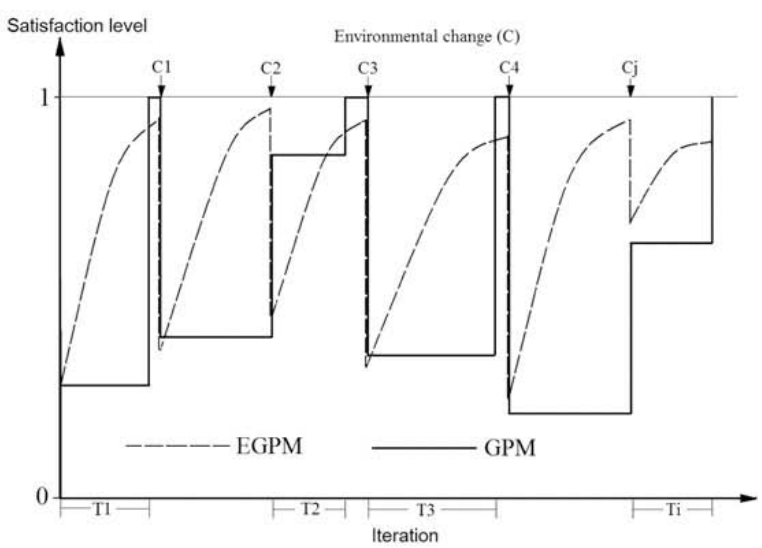

(b) dynamic environment

Fig. 7. Satisfaction level of demand in static environment and in dynamic environment. 


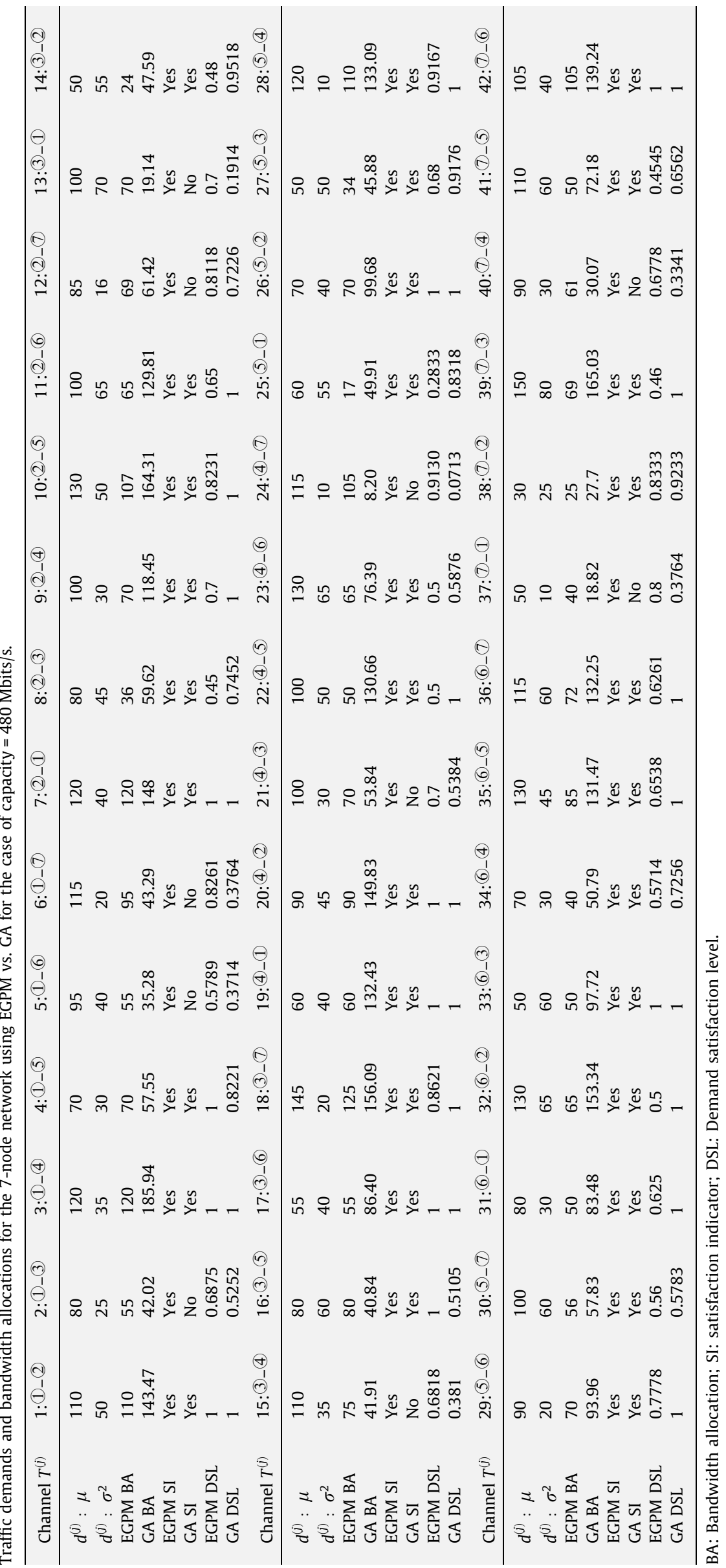


Fig. 7b shows an example of a changing satisfaction level in a dynamic environment. As can be seen in the figure, because the environment changes soon after or even before GPM reaches equilibrium, the allocation by GPM is effective only for a short pinch of time. On the other hand, the allocation by EGPM adapts to the changing environment more responsively.

\subsection{Comparison with genetic algorithm}

Here we compare the experimental results of EGPM with that of a genetic algorithm for the same problem of bandwidth allocation which can be found in [14]. Table 7 shows the results for the 7-node network with links running at $480 \mathrm{Mbits} / \mathrm{s}$. The experimental results of GA come from [14] and those of EGPM come from our own experiments.

As shown in Table 7, 10 (out of 42) channels failed to be satisfied using GA, whereas the EGPM algorithm satisfies all the channel demands. The average demand satisfaction levels of EGPM and GA are 0.7449 and 0.7890 , respectively; EGPM is slightly below because it gives preference to the SI index. In fact, EGPM tries to maximize all three objectives: DSL, BUL and fairness.

As shown in Table 8, EGPM is several orders of magnitude faster than GA in computing the solutions. And the convergence time of EGPM increases more steadily with the problem scale than GA.

Other than genetic algorithm, the comparison Lagrangian multiplier approach, the max-min algorithm, and the ant colony algorithm are also popular choices for solving the bandwidth allocation problem. We give a succinct comparison of these different approaches with EGPM/ GPM in Table 9.

\section{Conclusion}

In this paper, an approach based on the economic generalized particle model (EGPM) for intelligent allocation of network bandwidth is proposed. The approach transforms the complicated network bandwidth allocation problem into efficient, parallel allocation of network bandwidth. The mechanism is based on asynchronous modulation of prices of network bandwidth. The special features of the proposed model and its algorithm include: (1) high parallelism and real-time computational performance; (2) a market-oriented mechanism between the demands and service; (3) the microscopic characterization of an individual link and the macroscopic properties of the whole network being combined to achieve both fairness and efficiency; and (4) better adaptation to the real-time dynamic network environment.

The EGPM method can be applied to other optimization problems, such as resource allocation in grid, cache optimization in $\mathrm{CDN}$, etc. When solving grid computing problems by the EGPM algorithm, because the problem model of grid will be simpler than that of bandwidth allocation, only two of the ordinal numbers $i, j, k$ in the of bandwidth allocation model will be needed for the model of grid; moreover, the logic variable $x_{i k}^{(j)}$ will always be equal to 1 , and can be left out.

Table 8

Convergence times and iterations of EGPM vs. GA.

\begin{tabular}{|c|c|c|c|c|c|c|c|c|c|c|c|c|c|}
\hline \multicolumn{2}{|l|}{ Scale } & \multicolumn{4}{|c|}{ EGPM: 4 parallel Nodes } & \multicolumn{4}{|c|}{ EGPM: sequential } & \multicolumn{4}{|l|}{ GA } \\
\hline Nodes & Channels & Iterations & $\begin{array}{l}\text { Increase } \\
(\%)\end{array}$ & Time & $\begin{array}{l}\text { Increase } \\
(\%)\end{array}$ & Iterations & $\begin{array}{l}\text { Increase } \\
(\%)\end{array}$ & Time & $\begin{array}{l}\text { Increase } \\
(\%)\end{array}$ & Iterations & $\begin{array}{l}\text { Increase } \\
(\%)\end{array}$ & Time & $\begin{array}{l}\text { Increase } \\
(\%)\end{array}$ \\
\hline 4 & 12 & 134 & & 0.175 & & 103 & & 0.008 & & 2000 & & 1353 & \\
\hline 5 & 20 & 150 & 11.9 & 0.2 & 14.3 & 112 & 8.7 & 0.018 & 125.0 & 2000 & 0.0 & 3024 & 123.5 \\
\hline 6 & 30 & 171 & 14.0 & 0.245 & 22.5 & 130 & 16.1 & 0.029 & 61.1 & 2000 & 0.0 & 4761 & 57.4 \\
\hline 7 & 42 & 221 & 29.2 & 0.363 & 48.2 & 172 & 32.3 & 0.076 & 162.1 & 10,000 & 400.00 & 36971 & 676.5 \\
\hline
\end{tabular}

Table 9

EGPM vs. GPM and other well-known algorithms.

\begin{tabular}{|c|c|c|c|c|c|c|}
\hline & EGPM & GPM & $\begin{array}{l}\text { Lagrangrian } \\
\text { multiplier }\end{array}$ & Ant colony & Max-min & GA \\
\hline Flow control & $\begin{array}{l}\text { Decentralized/ } \\
\text { adaptive }\end{array}$ & $\begin{array}{l}\text { Decentralized/ } \\
\text { adaptive }\end{array}$ & Centralized/static & $\begin{array}{l}\text { Decentralized/ } \\
\text { adaptive }\end{array}$ & Centralized/static & $\begin{array}{l}\text { Decentralized/ } \\
\text { adaptive }\end{array}$ \\
\hline $\begin{array}{c}\text { Adaptive to } \\
\text { topology } \\
\text { changes }\end{array}$ & Fast & Middle & Slow & Middle & Fast & Middle \\
\hline $\begin{array}{l}\text { Routing } \\
\quad \text { overhead }\end{array}$ & Low & Low & High & Low & Low & Low \\
\hline $\begin{array}{l}\text { Routing } \\
\text { preference }\end{array}$ & $\begin{array}{l}\text { By Excess demand } \\
\text { function }\end{array}$ & $\begin{array}{l}\text { By hybrid energy } \\
\text { functions }\end{array}$ & By utility function & By pheromone laid & $\begin{array}{l}\text { By transmission } \\
\text { time/delay }\end{array}$ & By chromosomes \\
\hline $\begin{array}{l}\text { Information } \\
\text { exchange }\end{array}$ & $\begin{array}{l}\text { Can by } \\
\text { piggybacked in } \\
\text { data packets }\end{array}$ & $\begin{array}{l}\text { Can by } \\
\text { piggybacked in } \\
\text { data packets }\end{array}$ & $\begin{array}{l}\text { Separate routing } \\
\text { entries } \\
\text { transmission }\end{array}$ & $\begin{array}{l}\text { Can by } \\
\text { piggybacked in } \\
\text { data packets }\end{array}$ & $\begin{array}{l}\text { Separate routing } \\
\text { entries } \\
\text { transmission }\end{array}$ & $\begin{array}{l}\text { Can by } \\
\text { piggybacked in } \\
\text { data packets }\end{array}$ \\
\hline
\end{tabular}


In the future, we plan to improve EGPM so that it will become a generic algorithm for NP-hard combinatorial optimization problems, such as the traveling salesman problem (TSP), the quadratic assignment problem (QAP), etc., as well as for multi-objective optimization problems. We hope to develop the EGPM algorithm by a new approach, giving it the ability to deal with difficult problems that feature non-homogeneous, noisy, incomplete or obscured information, constrained resources, and massive processing of large amounts of data.

Here are some specific suggestions on future work.

1. The choices of coefficients in the EGPM algorithm and their impact should be further studied.

2. Communication times among computing entities should be reduced to improve the parallel execution of EGPM.

3. The present approach treats all demands to be equally important and tries to maximize the overall satisfaction. To support true QoS, priority classes with preferential treatment by the allocation mechanism need to be introduced. How to prove that the guarantee of a certain QoS can be met if certain conditions are satisfied "in theory" will be a key issue.

4. Our work in this paper is predominantly theoretical and based on a number of simplifying assumptions. Between this and a practical solution for real-life deployment, much work needs to be done.

5. Capacity planning including priority classes and reservation is not addressed in the current design of EGPM, which however is very important in practical bandwidth allocation.

\section{Acknowledgements}

This work was Supported by the National Science Foundation of China under Grant No. 60905043, the General Research Fund of Hong Kong Research Grant Council under Grant No. 7137/08E.

\section{Appendix A. Convergence proofs}

\section{A.1. Proof of Lemma 1}

Observe that when $\mu_{t} \leqslant 1$,

$\mu_{t} \cdot p^{*} \cdot z(p) \geqslant\left\|\mu_{t} \cdot z(p)\right\|^{2}$.

This can be seen by multiplying both sides of $p^{*} \cdot z(p) \geqslant$ $\|z(p)\|^{2}$ with $\mu_{t}^{2}$ and noticing that $\mu_{t}^{2} \cdot p^{*} \cdot z(p) \leqslant \mu_{t} \cdot p^{*}$. $z(p)$ because $\mu_{t} \leqslant 1$. From Eq. (14) and Walras' law we have

$$
\begin{aligned}
\left\|p(t+1)-p^{*}\right\|^{2}= & \left\|p(t)+\mu_{t} z(p(t))-p^{*}\right\|^{2} \\
= & \left\|\mu_{t} z(p(t))\right\|^{2}-2 \mu_{t} z(p(t)) \cdot p^{*}+\| p(t) \\
& -p^{*} \|^{2} \\
\leqslant & \left\|p(t)-p^{*}\right\|^{2} .
\end{aligned}
$$

Note that $p(t)$ belongs to $B\left(p^{*}, r\right)$ for all $t=0,1, \ldots$, when $p(0) \in B\left(p^{*}, r\right)$. Therefore, the sequence $\left\{\left\|p(t)-p^{*}\right\|\right\}_{t}$ con- verges, and as a result, the sequence $\{\|p(t)\|\}_{t}$ is bounded. From Walras' law it follows that

$\|p(t)\|^{2}=\|p(0)\|^{2}+\sum_{i=0}^{t-1} \mu_{i}^{2}\|z(p(i))\|^{2}$,

so that $\{\|p(t)\|\}_{t}$ is a growing and bounded sequence and hence convergent. The iteration formula yields

$p(t)=p(0)+\sum_{i=0}^{t-1} \mu_{i} z(p(i))$.

Hence, $\left\|p(0)+\sum_{i=0}^{t-1} \mu_{i} z(p(i))\right\|$ converges, too. From the triangular inequality we get

$$
\begin{aligned}
& \left\|p(0)+\sum_{i=0}^{t+l} \mu_{i} z(p(i))\right\| \\
& \geqslant\|\| p(0)+\sum_{i=0}^{t} \mu_{i} z(p(i))\|-\| \sum_{i=t+1}^{t+l} \mu_{i} z(p(i))\|\|
\end{aligned}
$$

and

$$
\|p(t+l)-p(t)\|=\left\|\sum_{i=t+1}^{t+l} \mu_{i} z(p(i))\right\| \rightarrow 0,
$$

when $t \rightarrow \infty$ and $l \geqslant 1$. Thus, $\{p(t)\}_{t}$ is a Cauchy sequence and hence convergent. Let $\widetilde{p}$ denote the limit point of this Cauchy sequence.

We now show that when $0<\mu_{L} \leqslant \mu_{t}$ the sequence $\{p(t)\}_{t}$ converges to the solution of $z(p)=0$. By setting $l=1$ it follows from Eq. (15) that $\mu_{t}\|z(p(t))\| \rightarrow 0$. Because $\mu_{L}\|z(p(t))\| \leqslant \mu_{t}\|z(p(t))\|$ holds and $z$ is continuous, $\widetilde{p}$ is a solution of $z(p)=0$.

\section{A.2. Proof of Lemma 2}

If Eq. (12) holds for $\alpha>0$ then it holds for any $\widetilde{\alpha}>\alpha$. Specifically, we can choose $\widetilde{\alpha}>0$ such that Eq. (12) holds for $p^{*}=\widetilde{\alpha} \widetilde{p}-2 \widetilde{p}$ instead of $\alpha \widetilde{p}$. Moreover we can take $\alpha$ such that $\|z(p)\|^{2}<2 \alpha z(p) \tilde{p}$ if $p$ is not a solution. We can deduce that $\left\|p(t+1)-p^{*}\right\|^{2}<\left\|p(t)-p^{*}\right\|^{2}$, and $\| p(t+1)-$ $\alpha \widetilde{p}\left\|^{2}<\right\| p(t)-\alpha \widetilde{p} \|^{2}$ when $p(t)$ is not a solution. By the parallelogram law we get

$\|p(t)-\alpha \widetilde{p}\|^{2}+\left\|p(t)-p^{*}\right\|^{2}=2\|p(t)-\widetilde{p}\|^{2}+2(\alpha-1)\|\widetilde{p}\|^{2}$.

By rearranging the terms we have

$$
\begin{aligned}
2\|p(t)-\widetilde{p}\|^{2}= & 2(\alpha-1)\|\widetilde{p}\|^{2}-\|p(t)-\alpha \widetilde{p}\|^{2}-\left\|p(t)-p^{*}\right\|^{2} \\
> & 2(\alpha-1)\|\widetilde{p}\|^{2}-\|p(t+1)-\alpha \widetilde{p}\|^{2} \\
& -\left\|p(t+1)-p^{*}\right\|^{2}=2\left\|p(t+1)-p^{*}\right\|
\end{aligned}
$$

and hence $\{p(t)\}_{t}$ converges monotonically to $\widetilde{p}$.

\section{A.3. Proof of Lemma 3}

Let $z$ satisfy $\mathrm{C} 2$ on $E_{\bar{\zeta}}=\left\{p \in \mathbb{R}_{+}^{n}:\|z(p)\|<\bar{\varsigma}\right\}$ with constant $\bar{\sigma}$. By the homogeneity of excess demand we know that $\hat{z}$ obtains all its values on the unit simplex $\Delta=$ $\left\{p \in \mathbb{R}_{+}^{n}: \sum_{j=1}^{J} \sum_{k=1}^{n_{j}} P_{k}^{(j)}=1\right\}$. Because of P4 and P5 it can be seen that $p^{*} \cdot z(p(t)) \rightarrow \infty$ when $p(t) \rightarrow p$ and $J K_{p} \neq \emptyset$. As a result, we have 
$\lim _{p(t) \rightarrow p} p^{*} \cdot \hat{z}(p)>0$

when $J K_{p} \neq \emptyset$. From this property, the continuity property, and $\mathrm{C} 1$, it follows that there exists $\delta>0$ such that $p^{*} \cdot \hat{z}(p) \geqslant \delta$ for all $p \in \Delta \backslash S$, where $S=\left\{p \in \Delta: p_{k}^{(j)}>\right.$ $\varsigma^{\prime}, \forall j=\overline{1, J}, k=\overline{1, n_{p}}, \varsigma^{\prime}>0$ is chosen such that $E_{\bar{\zeta}} \cap \Delta \subset S$.

Clearly, the infimum of $p^{*} \cdot \hat{z}(p)$ over $S \backslash E_{\bar{\zeta}}$ is positive, since otherwise $\hat{z}$ would violate $C 1$. Let $\alpha>0$ denote this infimum. We have $p^{*} \hat{z}(p) \geqslant \min \{\delta, \alpha\}$ for all $p \in \Delta \backslash E_{\bar{\zeta}}$. Because $\|\hat{z}(p)\| \leqslant M$ we get $p^{*} \cdot \hat{z}(p) \geqslant \hat{\sigma}\|\hat{z}(p)\|^{2}$ for all $p \in \Delta \backslash E_{\bar{\zeta}}$ by choosing $\hat{\sigma}<\min \{\delta, \alpha\} \backslash M^{2}$. The result follows by setting $\sigma=\min \{\bar{\sigma}, \hat{\sigma}\}$.

\section{A.4. Proof of Theorem 1}

The process of Eq. (11) can be expressed as

$p(t+1)=p(t)+\lambda_{t} \hat{z}(p(t))$

where $\lambda_{t}=\min \left\{\gamma_{t}, 1\right\}$, and $\hat{z}$ is as defined in Lemma 3 . When $z$ has the properties P1-P4, so does $\hat{z}$, and P5 implies that $\hat{z}$ has the following property

$\mathrm{P5}^{\prime}: \lim _{p(t) \rightarrow p}\left[\max _{j, k \in J K_{p}} \hat{z}_{j}(p(t))\right]>0, \quad$ when $p \neq 0$, and

$J K_{p}=\left\{j, k: p_{k}^{(j)}=0\right\} \neq \emptyset$.

Moreover, we know from Lemma 3 that $p^{*} \cdot \hat{z}(p) \geqslant$ $\sigma\|\hat{z}(p)\|^{2}$ holds for all $p>0$ when $z$ satisfies C1-C2. Due to homogeneity $p^{*}$ can be replaced by $p^{*} / \sigma$ in $\mathrm{C} 1$ and $\mathrm{C} 2$; hence, without loss of generality we may suppose that $\sigma=1$. It follows then from Lemma 1 that the iteration converges.

Let us show that due to $\mathrm{P}^{\prime}$ the parameter $\lambda_{t}$ is updated only finitely many times, from which it follows that $\lambda_{t}$ has a positive lower bound that is required by Lemma 1 to obtain convergence to a solution of Eq. (7). On the contrary, suppose that $\lambda_{t} \rightarrow 0$. It then follows that $p_{t} \rightarrow p$, where some components of $p$ are zero, i.e., $J K_{p} \neq \emptyset$. Namely, if the sequence $\{p(t)\}_{t}, p(t)>0$ for all $t$, converges to some point in $\mathbb{R}_{+}^{n}$, then the parameters $\gamma_{t}$ and $\lambda_{t}$ would be updated only finitely many times and $\lambda_{t}$ could not converge to zero. Hence, we have $p_{k}^{(j)}(t) \rightarrow 0$ for all $j, k \in J K_{p}$, i.e., for all $\varsigma>0$ we find $N_{\varsigma} \geqslant 0$ such that $p_{k}^{(j)}(t)<\varsigma$, when $t \geqslant N_{\varsigma}$ and $j, k \in J K_{p}$. Thus, by the continuity property and P5' there are $l, h \in J K_{p}$ and $N \geqslant 0$ such that $p_{h}^{(l)} \rightarrow 0$, and $z_{l}(p(t))>0$ for all $t \geqslant N$. Now we get from the iteration formula that $p_{h}^{(l)}(t+1)>p_{h}^{(l)}(t)$ for all $t \geqslant N$, which contradicts $p_{h}^{(l)} \rightarrow 0$. Hence, $\lambda_{t}$ is updated finitely many times, i.e., there exists $\bar{N}$ such that $\lambda_{t}=\lambda_{\bar{N}}$ for all $t \geqslant \bar{N}$. Convergence to a solution of Eq. (7) follows from Lemma 1.

Let us assume that there is a unique ray of solutions for Eq. (7). Then the process of Eq. (11) converges to a point $\tilde{p}=\beta p^{*}$, where $\beta>0$. From Lemma 3 we see that there exists $\alpha>0$ such that for $\alpha \tilde{p}$ we have $2 \alpha \tilde{p} \cdot \hat{z}(p) \geqslant\|\hat{z}(p)\|^{2}$ for all $p>0$. Lemma 2 then implies monotonical convergence.

\section{References}

[1] D. Shuai, X. Feng, The parallel optimization of network bandwidth allocation based on generalized particle model, Computer Networks 50 (9) (2006) 1219-1246.
[2] D. Bertsekas, R. Gallager, Data Networks, Prentice-Hall, New Jersey, 1992.

[3] F.P. Kelly, Charging and rate control for elastic traffic, European Transactions on Telecommunications 29 (1997) 1009-1016.

[4] F.P. Kelly, A. Maulloo, D. Tan, Rate control in communication networks: shadow prices, proportional fairness and stability, Journal of the Operational Research Society 49 (1998) 237-252.

[5] F.P. Kelly, Mathematical modeling of the Internet, in: B. Engquist, W. Schmid (Eds.), Mathematics Unlimited, Springer-Verlag, Berlin, 2001, pp. 685-702.

[6] S.H. Low, A duality model of TCP and queue management algorithms, IEEE ACM Transactions on Networking 11 (4) (2003) 525-536.

[7] S.H. Low, F. Paganini, L. Wang, J.C. Doyle, Linear stability of TCP/RED and a scalable control, Computer Networks 43 (5) (2003) 633647.

[8] S.H. Low, F. Paganini, J.C. Doyle, Internet congestion control, IEEE Control Systems Magazine 22 (2) (2002) 28-43.

[9] S.H. Low, L. Peterson, L. Wang, Understanding vegas: a duality model, Journal of ACM 49 (2) (2002) 207-235.

[10] M. Dorigo, C. Blum, Ant colony optimization theory: a survey, Theoretical Computer Science 344 (2005) 243-278.

[11] S.H. Ngo, X.H. Jiang, S. Horiguchi, An ant-based approach for dynamic RWA in optical WDM networks, Photonic Network Communications 11 (2006) 39-48.

[12] E. Bonabeau, M. Dorigo, G. Theraulaz, Inspiration for optimization from social insect behavior, Nature 406 (6791) (2000) 39-42.

[13] S. Forrest, Genetic algorithms-principles of natural selection applied to computation, Science 261 (5123) (1993) 872-878.

[14] A. Pitsillides, G. Stylianou, C.S. Pattichis, A. Sekercioglu, A. Vasilakos, Bandwidth allocation for virtual paths (BAVP): investigation of performance of classical constrained and genetic algorithm based optimization techniques, in: Proc. of INFOCOM, vol. 3, 2000, pp. 1501-1510.

[15] A. Pitsillides, G. Stylianou, C.S. Pattichis, A. Sekercioglu, A. Vasilakos, Aggregated bandwidth allocation: investigation of performance of classical constrained and genetic algorithm based optimization techniques, Computer Communications 25 (16) (2002) 1443-1453.

[16] X. Wang, H. Schulzrinne, Pricing network resources for adaptive applications in a differentiated services network, in: Proc. of INFOCOM, vol. 2, 2001, pp. 943-952.

[17] M. Baglietto, R. Bolla, F. Davoli, M. Marchese, M. Mongelli, A proposal of new price-based call admission control rules for guaranteed performance services multiplexed with best effort traffic, Computer Communications 26 (13) (2003) 1470-1483.

[18] N. Jin, S. Jordan, The effect of bandwidth and buffer pricing on resource allocation and QoS, Computer Networks 46 (1) (2004) 5371.

[19] M. Bitsaki, G.D. Stamoulis, C. Courcoubetis, Auction-based bandwidth trading in a competitive hierarchical market, in: Proc. IEEE International Conference on Next Generation Internet Networks, vol. 372-379, 2005.

[20] R. Wolski, J.S. Plank, J. Brevik, T. Bryan, Analyzing market-based resource allocation strategies for the computational grid, International Journal of High Performance Computing Applications 15 (3) (2005) 258-281.

[21] P. Thomas, D. Teneketzis, J.K. Mackie-Mason, A market-based approach to optimal resource allocation in integrated-services connection-oriented networks, Operations Research 50 (4) (2002) 603-616.

[22] V.A. Siris, Resource Control for elastic traffic in CDMA networks, in: Proc. of the 8th Annual International Conference on Mobile Computing and Networking, Atlanta, USA, 2002, pp. 193-204.

[23] S. Netessine, R. Shumsky, Introduction to the theory and practice of yield management, INFORMS Transactions on Education 3 (1) (2002) 34-44.

[24] R.M. Starr, General Equilibrium Theory-An Introduction, Cambridge University Press, 1997.

[25] X. Feng, F.C.M. Lau, D. Shuai, A new generalized particle approach to parallel bandwidth allocation, Computer Communications 29 (18) (2006) 3933-3945.

[26] R. Jain, D. Chiu, W. Hawe, A quantitative measure of fairness and discrimination for resource allocation in shared computer systems, DEC Research Report TR-301, September 1984.

[27] J.-Y. Le Boudec, Rate adaptation, congestion control and fairness: a tutorial, 2005. <http://citeseer.ist.psu.edu/boudec00rate.html>.

[28] A. Mas-Colell, M.D. Whinston, J.R. Green, Microeconomic Theory, Oxford University Press, New York, 1995.

[29] P.A. Samuelson, Foundations of Economic Analysis, Harvard University Press, Cambridge, MA, 1947. 


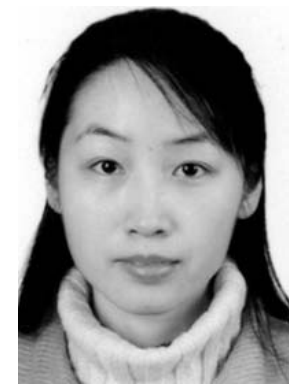

Xiang Feng received the M.Sc. degree in System Engineering from Naval Engineering University, Wuhan, in 2003. She received the Ph.D. degree in Control Theory and Engineering from East China University of Science and Technology, Shanghai, in 2006. She worked as a postdoctoral fellow in the Department of Computer Science of the University of Hong Kong from 2006 to 2008 . She is presently an associate professor of the Department of Computer Science and Engineering, East China University of Science and Technology. Her research interests include mechanics-related nature-inspired algorithm, parallel and distributed computing and computer networks.

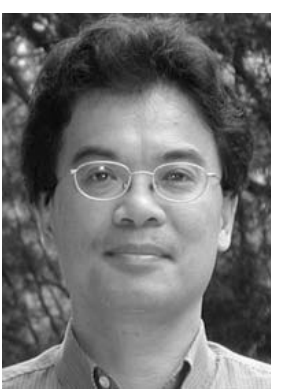

Francis C.M. Lau received the PhD degree in computer science from the University of Waterloo in 1986. He has been a faculty member in the Department of Computer Science of the University of Hong Kong since 1987, and served as the head of department from 2000 to 2005. He received a Golden Core recognition in 1998 and an IEEE Third Millennium Medal in 2000 for outstanding achievements and contributions to the IEEE Computer Society. Prof. Lau's research interests include parallel and distributed computing, mobile and pervasive computing, and computer art and music. 